\title{
Synergies between the constitutive relation error concept and PGD model reduction for simplified V\&V procedures
}

\author{
Ludovic Chamoin*, Pierre-Eric Allier and Basile Marchand
}

"Correspondence: chamoin@Imt.ens-cachan.fr LMT, ENS Cachan, CNRS,

Université Paris Saclay, 61 Avenue du Président Wilson, 94235 Cachan, France

\begin{abstract}
The paper deals with the constitutive relation error (CRE) concept which has been widely used over the last 40 years for verification and validation of computational mechanics models. It more specifically focuses on the beneficial use of model reduction based on proper generalized decomposition (PGD) into this CRE concept. Indeed, it is shown that a PGD formulation can facilitate the construction of so-called admissible fields which is a technical key-point of CRE. Numerical illustrations, addressing both model verification and model updating, are presented to assess the performances of the proposed approach.
\end{abstract}

Keywords: Error estimation, Model updating, Model reduction, Constitutive relation error (CRE), Proper generalized decomposition (PGD)

\section{Background}

Mathematical models and their solutions, either analytical or numerical, are fundamental in science and engineering activities as they constitute the basic ingredient of simulations that enable to predict the behavior of physical phenomena. Consequently, a permanent issue is the verification and validation of these models, which nowadays can attain very high levels of complexity, in order to certify the quality of numerical simulations. On the one hand, verification deals with the assessment of the numerical (FE) model with respect to initial mathematical model, and implies the estimation of discretization error in order to control the quality of the approximate numerical solution. In this context, a large set of a posteriori error estimates has appeared over the last thirty years (see [1-3] for an overview). On the other hand, validation addresses the capability of mathematical models to represent a faithful abstraction of the real (physical) world. It aims at identifying or updating model parameters in order to minimize the discrepancy between numerical predictions and experimental measurements, and leads to the solution of inverse problems [4].

In the context of model verification and validation, and particularly for computational mechanics models in which the constitutive relation is a major component, the constitutive relation error (CRE) concept is a convenient and powerful tool. The idea of CRE is rather simple: so-called admissible fields verifying all equations of the model except the constitutive relation are constructed, then the residual associated with the constitutive relation is measured. The CRE concept was first introduced as a robust a posteriori

(c) The Author(s) 2016. This article is distributed under the terms of the Creative Commons Attribution 4.0 International License (http://creativecommons.org/licenses/by/4.0/), which permits unrestricted use, distribution, and reproduction in any medium, provided you give appropriate credit to the original author(s) and the source, provide a link to the Creative Commons license, and indicate if changes were made. 
error estimator in FE computations [5], enabling to compute both strict and effective discretization error bounds for linear and more generally convex structural mechanics problems, and to lead mesh adaptivity processes. It was primarily used for linear thermal and elasticity problems [6,7] before being extended to nonlinear time dependent problems [8,9] and to goal-oriented error estimation [10-12]. The use of CRE for model verification, for which a general overview can be found in [2], requires in particular the computation of admissible dual fields which are fully equilibrated. This requirement, which is the main practical issue both in terms of computational cost and implementation technicality, was addressed by means several techniques that post-process the FE solution at hand $[2,6,13-20]$. During the 90s, the CRE concept was extended to model identification/updating. First introduced for dynamics models [21-24], this method was latter successfully used in many calibration applications including defects [25], uncertain measurements and behaviors [26,27], or corrupted measurements [28,29]. It was also used in the context of full-field measurements [30,31]. After initial studies in which measurements were included as additional admissibility constraints, a more flexible and effective strategy was developed. Denoted as modified CRE (mCRE), this strategy consists in relaxing constraints on measurements and other uncertain data, proposing a general framework in which reliable theoretical and experimental information (equilibrium, sensor position,...) is favored to define admissibility spaces, and residual on complementary information (material behavior, sensor measurements,...) is measured. It acts in an iterative two-steps algorithm, in which optimal admissible fields are first computed, before minimizing the obtained mCRE functional with respect to model parameters. The use of mCRE presents interesting advantages; it has excellent capacities to localize structural defects spatially, it is very robust with respect to noisy measurements, and it has good convexity properties.

The objective of the paper is to present new numerical tools, based on model reduction techniques and offline-online strategy, that can be coupled to the CRE concept to make this latter fully implementable and exploitable for practical industrial applications. They particularly aim at decreasing the computational cost and technicality level which are required when computing admissible fields, leading to fast and inexpensive verification and validation $(V \& V)$ procedures. For that purpose, we decide to refer to the proper generalized decomposition (PGD) which is an a priori model reduction technique that has been extensively used over the last decade to solve multi-parametric problems (see [3234]). Consider a general linear $D$-dimensional problem of the form:

$$
\mathcal{L} u=g, \quad u \in \mathcal{X}=\mathcal{X}_{1} \otimes \mathcal{X}_{2} \otimes \cdots \otimes \mathcal{X}_{D}
$$

where $\mathcal{L}$ is an operator defined on the tensor space $\mathcal{X}$. PGD is a low-rank tensor method that consists in searching an approximation of $u$ in a low-dimensional tensor subspace of $\mathcal{X}_{m} \subset \mathcal{X}$ made of canonical format tensors of rank $m$ :

$$
u_{m}=\sum_{i=1}^{m} w_{i}^{1} \otimes w_{i}^{2} \cdots \otimes w_{i}^{D}, \quad w_{i}^{\mu} \in \mathcal{X}_{\mu}
$$

Among the various strategies to construct $u_{m}$ [35], we focus on the one called progressive Galerkin. Introducing the global weak formulation of the problem:

Find $u \in \mathcal{X}$ such that $B(u, v)=F(v) \quad \forall v \in \mathcal{X}$ 
and assuming that the rank $m-1$ decomposition $u_{m-1}$ is known, the rank $m$ decomposition $u_{m}=u_{m-1}+w^{1} \otimes w^{2} \cdots \otimes w^{D}$ is searched such that:

$$
\begin{aligned}
& B\left(u_{m}, \delta v\right)=F(\delta v) \\
& \quad \forall \delta v=\delta w^{1} \otimes w^{2} \cdots \otimes w^{D}+w^{1} \otimes \delta w^{2} \cdots \otimes w^{D}+\cdots+w^{1} \otimes w^{2} \cdots \otimes \delta w^{D}
\end{aligned}
$$

with $\delta w^{\mu} \in \mathcal{X}_{\mu}$. This formulation naturally leads to a nonlinear problem where a set of coupled low-dimensional problems has to be solved:

$$
\begin{aligned}
B\left(w^{1} \otimes w^{2} \cdots \otimes w^{D}, \delta w^{1} \otimes w^{2} \cdots \otimes w^{D}\right) & =R_{m-1}\left(\delta w^{1} \otimes w^{2} \cdots \otimes w^{D}\right) & & \forall \delta w^{1} \in \mathcal{X}_{1} \\
B\left(w^{1} \otimes w^{2} \cdots \otimes w^{D}, w^{1} \otimes \delta w^{2} \cdots \otimes w^{D}\right) & =R_{m-1}\left(w^{1} \otimes \delta w^{2} \cdots \otimes w^{D}\right) & & \forall \delta w^{2} \in \mathcal{X}_{2} \\
\vdots & =\vdots & & \\
B\left(w^{1} \otimes w^{2} \cdots \otimes w^{D}, w^{1} \otimes w^{2} \cdots \otimes \delta w^{D}\right) & =R_{m-1}\left(w^{1} \otimes w^{2} \cdots \otimes \delta w^{D}\right) & & \forall \delta w^{D} \in \mathcal{X}_{D}
\end{aligned}
$$

with $R_{m-1}(v)=F(v)-B\left(u_{m-1}, v\right)$. This problem is in practice solved with an iterative (fixed point) strategy.

On the one hand, in the context of model verification, the CRE concept was already used to control PGD approximations (see a posteriori error estimates developed in [36,37]) or to directly drive the PGD process with CRE minimization [38]. Nevertheless, the use of PGD in CRE implementation has never been investigated and we wish to show here that there are major advantages to do so, in particular for the construction of equilibrated fields. On the other hand, in the context of model validation, PGD was used for model updating within classical procedures with least square minimization [39]. It was also recently used in particular applications involving robust model updating with the CRE concept $[40,41]$. Here, the goal is to give a general framework on the effective use of PGD for model updating with CRE. For the sake of simplicity and clarity, we consider scalar linear elliptic (stationary thermal) problems even though extensions to elasticity or more complex problems (nonlinear or transient analyses), briefly addressed in this paper, are possible with regards to existing literature $[2,8]$.

The paper outline is as follows: after presenting the mathematical model of interest in "Reference problem and approximate FE solution" section, the CRE concept is reviewed in "Basics on the CRE concept" section; its extension to model validation with the mCRE concept is addressed in "Extension of the CRE concept for model updating: modified CRE" section; the use of PGD in addition to CRE for the construction of admissible fields is shown in details in "Coupling PGD with CRE in model verification" section for model verification, and in "Coupling PGD with mCRE in model validation" section for model validation; illustrative numerical results are reported in "Results and discussion" section; conclusions are drawn in "Conclusions" section.

\section{Methods}

\section{Reference problem and approximate FE solution}

We consider a steady-state thermal problem that consists in finding the temperature/flux pair $(u, \boldsymbol{q})$ such that:

$$
\begin{array}{lll} 
& u=0 \text { on } \Gamma_{D} & \text { (kinematic constraints) } \\
-\nabla \cdot \boldsymbol{q}=f \text { in } \Omega ; & \boldsymbol{q} \cdot \boldsymbol{n}=g \text { on } \Gamma_{N} & \text { (balance equations) } \\
& \boldsymbol{q}=\mathcal{K} \nabla u & \text { (constitutive relation) }
\end{array}
$$


$\Omega$ is an open bounded subset of $\mathbb{R}^{d}$ with Lipschitz boundary $\partial \Omega$, and $\Gamma_{D}$ and $\Gamma_{N}$ are complementary parts of $\partial \Omega$ such that $\overline{\Gamma_{D} \cup \Gamma_{N}}=\partial \Omega, \Gamma_{D} \cap \Gamma_{N}=\emptyset$, and $\left|\Gamma_{D}\right| \neq 0$. We assume that $f \in L^{2}(\Omega)$ and $\mathcal{K} \in\left[L^{\infty}(\Omega)\right]^{d \times d}$ is a symmetric, uniformly bounded and positive matrix in the sense that there exists $k_{\max } \geq k_{\min }>0$ such that

$$
\forall \xi \in \mathbb{R}^{d}, \quad k_{\min }|\xi|^{2} \leq \mathcal{K} \xi \cdot \xi \leq k_{\max }|\xi|^{2} \quad \text { a.e. in } \Omega .
$$

Considering the Hilbert space $\mathcal{U}=\left\{v \in H^{1}(\Omega), v=0\right.$ on $\left.\Gamma_{D}\right\}$ equipped with the $H^{1}$-norm $\|v\|_{1}$, the weak formulation of (6) reads:

$$
\text { Find } u \in \mathcal{U} \text { such that } a(u, v)=l(v) \quad \forall v \in \mathcal{U}
$$

with

$$
a(u, v)=\int_{\Omega} \mathcal{K} \nabla u \cdot \nabla v, \quad l(v)=\int_{\Omega} f v+\int_{\Gamma_{N}} g v .
$$

The bilinear form $a$ is symmetric, continuous and coercive on $\mathcal{U}$. It hence defines an inner product and induces the energy norm $\|v\|_{\mathcal{U}}=\sqrt{a(v, v)}$ which is equivalent to $\|v\|_{1}$ on $\mathcal{U}$. We also denote $\|v\|_{0}$ the $L^{2}$-norm on $\mathcal{U}$. Existence and uniqueness of the solution $u$ to (8) is provided by the Lax-Milgram theorem. We note that (8) is equivalent to the minimization of the potential energy $J_{1}(v)=\frac{1}{2} a(v, v)-l(v)$ on $\mathcal{U}$.

Let $\mathcal{T}_{h}$ be a regular (non-degenerate) partition of $\Omega$. Introducing the space $\mathcal{U}_{h}$ of continuous and locally supported functions which are polynomials on each element $K \in \mathcal{T}_{h}$, the conforming FE approximation of (8) reads:

$$
\text { Find } u_{h} \in \mathcal{U}_{h} \text { such that } a\left(u_{h}, v\right)=l(v) \quad \forall v \in \mathcal{U}_{h}
$$

We thus define the discretization error $e=u_{h}-u \in \mathcal{U}$, for which a measure $\|e\|_{\|}$in terms of the energy norm can be introduced to express the global quality of the approximate solution $u_{h}$. Introducing the residual functional and associated dual norm:

$$
R(v)=l(v)-a\left(u_{h}, v\right) ; \quad\|R\|_{*}=\sup _{v \in \mathcal{U}, v \neq 0} \frac{|R(v)|}{\|v \mid\| \mathcal{U}}
$$

leads to $\|e\|\left\|_{\mathcal{U}}=\right\| R \|_{*}$. In the context of model verification, a main goal of a posteriori error estimators is to assess the value of $\|\mid\|_{\|} \mathcal{U}$.

\section{Basics on the CRE concept}

We present here the foundations and implementation of the CRE concept, built from a dual approach and measuring the residual on the constitutive relation $\boldsymbol{q}=\mathcal{K} \nabla u$, in the context of model verification.

Using the approximation approach with primal variational principle (10), that consists of minimizing the potential energy $J_{1}(v)$ on $\mathcal{U}_{h}$, leads to:

$$
\|\| e \|_{\mathcal{U}}^{2}=2\left[J_{1}\left(u_{h}\right)-J_{1}(u)\right] \geq 2\left[J_{1}\left(u_{h}\right)-J_{1}(v)\right] \quad \forall v \in \mathcal{U}_{h}
$$

This shows that any $v \in \mathcal{U}_{h}$ can only enable to compute a lower bound on the discretization error $\|\mid\|_{\|} \|_{\mathcal{u}}$; this lower bound is in practice usually poor unless $v$ is chosen suitably.

Getting an upper bound on $\|\mid\|_{\mathcal{U}}$ requires to use the complementary variational principle. Using the subspace $\mathcal{S}$ of $H(d i v, \Omega)=\left\{\pi \in\left[L^{2}(\Omega)\right]^{d}, \nabla \cdot \pi \in L^{2}(\Omega)\right\}$ defined as:

$$
\begin{aligned}
\mathcal{S} & =\left\{\boldsymbol{\pi} \in H(\operatorname{div}, \Omega), \nabla \cdot \boldsymbol{\pi}+f=0 \text { in } \Omega, \boldsymbol{\pi} \cdot \boldsymbol{n}=g \text { on } \Gamma_{N}\right\} \\
\Longleftrightarrow \mathcal{S} & =\left\{\boldsymbol{\pi} \in H(\operatorname{div}, \Omega), \int_{\Omega} \boldsymbol{\pi} \cdot \nabla v=\int_{\Omega} f v+\int_{\Gamma_{N}} g v \quad \forall v \in \mathcal{U}\right\}
\end{aligned}
$$


this complementary variational principle defines the solution flux field $\boldsymbol{q}=\mathcal{K} \nabla u$ as:

$$
\boldsymbol{q}=\arg \min _{\boldsymbol{\pi} \in \mathcal{S} J_{2}(\boldsymbol{\pi})} ; \quad J_{2}(\boldsymbol{\pi})=\frac{1}{2} \int_{\Omega} \mathcal{K}^{-1} \boldsymbol{\pi} \cdot \boldsymbol{\pi}=\frac{1}{2}\|\| \boldsymbol{\pi} \|_{\mathcal{S}}^{2}
$$

where $\|\mid \bullet\| \|_{\mathcal{S}}$ is the energy norm for flux fields. A direct consequence is:

$$
\|\| \boldsymbol{\pi}-\boldsymbol{q} \|_{\mathcal{S}}^{2}=2\left[J_{2}(\boldsymbol{\pi})-J_{2}(\boldsymbol{q})\right]
$$

Noticing that $J_{1}(u)=-J_{2}(\boldsymbol{q})$ leads to the property (Prager-Synge equality):

$$
\begin{aligned}
\|\| \|_{\mathcal{U}^{2}}^{2} & =2\left[J_{1}\left(u_{h}\right)+J_{2}(\boldsymbol{q})\right]=2\left[J_{1}\left(u_{h}\right)+J_{2}(\boldsymbol{\pi})\right]-\|\| \boldsymbol{\pi}-\boldsymbol{q} \|\left.\right|_{\mathcal{S}} ^{2} \\
& =2 E_{C R E}^{2}\left(u_{h}, \boldsymbol{\pi}\right)-\|\boldsymbol{\pi}-\boldsymbol{q}\|_{\mathcal{S}}^{2}
\end{aligned}
$$

where we introduced the CRE functional $E_{C R E}$ defined as:

$$
E_{C R E}^{2}(v, \pi)=J_{1}(v)+J_{2}(\pi)=\frac{1}{2}\|\mid \pi-\mathcal{K} \nabla v\| \|_{\mathcal{S}}^{2} \quad \forall(v, \pi) \in \mathcal{U} \times \mathcal{S}
$$

that measures the non-verification of the constitutive relation for any pair $(v, \pi) \in \mathcal{U} \times \mathcal{S}$. In the following, such a pair is referred as admissible: a field $v \in \mathcal{U}$ is said kinematically admissible (KA); a field $\pi \in \mathcal{S}$ (i.e. verifying balance equations exactly) is said statically admissible (SA). Using the CRE concept, the reference problem (6) can be formulated as:

$$
(u, \boldsymbol{q})=\underset{(v, \pi) \in \mathcal{U} \times \mathcal{S}}{\arg \min } E_{C R E}(v, \pi)
$$

Remark 1 For all material models described using internal variables and standard formulation, and introducing a suitable definition of admissibility spaces, a more general local (in space and time) expression of the CRE functional reads [2,8]:

$$
E_{C R E}^{2}(X, Y)=\phi(X)+\phi^{*}(Y)-\langle X, Y\rangle
$$

where $(X, Y)$ is a dual pair (with duality pairing $\langle X, Y\rangle$ ), and $\phi$ and $\phi^{*}$ are dual (in the Legendre Fenchel sense) convex (pseudo-) potentials related to free energy or dissipation. For the present case, $\phi(\nabla v)=\frac{1}{2} \mathcal{K} \nabla v \cdot \nabla v$ and $\phi^{*}(\pi)=\frac{1}{2} \mathcal{K}^{-1} \pi \cdot \pi$.

Consequently, and provided that a flux field $\pi \in \mathcal{S}$ is available, we observe from (16) that the term $\sqrt{2} E_{C R E}\left(u_{h}, \pi\right)$ is a computable upper bound on $\|\mid e\|_{\mathcal{U}}$. The quality of this bound depends on that of $\pi$.

The constraints in space $\mathcal{S}$ make the construction of SA solutions awkward. A first possibility, which is the most effective, would consist in using a FE discretization with equilibrium elements on the complementary problem (14) (dual approach, see [42-44]). However, this is in practice unrealistic as it would require the solution of an additional global problem, with large computational efforts and non-conventional FE spaces. In "Coupling PGD with CRE in model verification" section, we present the basis of a technique (referred as hybrid-flux or EET in the literature) that enables to compute a flux field $\widehat{\boldsymbol{q}}_{h} \in \mathcal{S}$ [and therefore the a posteriori error estimate $\left.\sqrt{2} E_{C R E}\left(u_{h}, \widehat{\boldsymbol{q}}_{h}\right)\right]$ from a postprocessing of the FE field $\boldsymbol{q}_{h}$ at hand. The PGD strategy will be used within this technique in order to facilitate implementation issues.

Remark 2 It can be shown that using the hybrid-flux (or EET) technique to construct an admissible flux field $\widehat{\boldsymbol{q}}_{h}$ enables to obtain a lower error bound from the CRE functional [2, 6]; it is of the form $E_{C R E}\left(u_{h}, \widehat{\boldsymbol{q}}_{h}\right) \leq C\|\| e \|_{\mathcal{U}}$, where $C$ is a constant independent of the mesh size, proving that the constructed error estimate has the same convergence rate as the true discretization error. 


\section{Extension of the CRE concept for model updating: modified CRE}

We now consider that the material operator $\mathcal{K}$ depends on a set $\boldsymbol{p} \in \mathcal{P}$ of parameters to be identified from experimental measurements. To solve the associated ill-posed inverse problem, we introduce the energy-based concept of modified constitutive relation error (mCRE) $[28,45]$, which can be seen as a direct extension of the CRE concept developed in the previous section. The mCRE functional, still based on duality between admissible primal and dual fields $(v, \pi) \in \mathcal{U} \times \mathcal{S}$, is defined as:

$$
\mathcal{E}_{m C R E}^{2}(v, \boldsymbol{\pi}, \boldsymbol{p}, \boldsymbol{s})=\frac{1}{2}\|\boldsymbol{\pi}-\mathcal{K}(\boldsymbol{p}) \nabla v\|_{\mathcal{S}}^{2}+\frac{1}{2} \frac{r}{1-r} \sigma\|\boldsymbol{\Pi} v-\boldsymbol{s}\|_{0}^{2}
$$

where $s$ is the set of experimental data, $\Pi$ is an extraction operator, $\sigma$ is a scaling coefficient, and $r \in[0,1]$. The two terms that compose the mCRE functional are modeling error term (i.e. classical CRE term) and measurement error term, respectively; these terms are weighted depending on the value of $r$.

Remark 3 The value of $r$ should generally be set in regards to the $a$ priori reliability on both model and measurements. For instance, the Morozov principle or L-curve method [46] may be used to define $r$ with respect to data noise. The influence of $r$ on the sensitivity with respect to measurement uncertainties, and therefore on the quality of the updating performed using mCRE, was illustrated in [47].

The solution of the inverse identification problem is then defined as the result of a double minimization:

$$
\boldsymbol{p}_{0}=\underset{\boldsymbol{p} \in \mathcal{P}}{\operatorname{argmin}}\left(\min _{(v, \pi) \in \mathcal{U} \times \mathcal{S}} \mathcal{E}_{m C R E}^{2}(v, \boldsymbol{\pi}, \boldsymbol{p}, \boldsymbol{s})\right)
$$

In practice, this problem is solved using an iterative alternated minimization procedure with fixed point method as detailed in the following algorithm:

1. Initialize the parameter set $\boldsymbol{p}_{0}$ and set iteration threshold $\epsilon$ Iteration loop

2. Compute $(\widehat{u}, \widehat{\boldsymbol{q}})=\arg \min _{(v, \pi) \in \mathcal{U} \times \mathcal{S}} \mathcal{E}_{m C R E}^{2}\left(v, \boldsymbol{\pi}, \boldsymbol{p}_{0}, \boldsymbol{s}\right)$

3. Define cost function $\mathcal{F}(\boldsymbol{p})=\mathcal{E}_{m C R E}^{2}(\widehat{u}, \widehat{\boldsymbol{q}}, \boldsymbol{p}, \boldsymbol{s})$

4. Minimize the cost function: $\boldsymbol{p}_{0}=\arg \min _{\boldsymbol{p} \in \mathcal{P}} \mathcal{F}(\boldsymbol{p})$

5. If $\mathcal{F}\left(\boldsymbol{p}_{0}\right) \leq \epsilon$, stop. Otherwise, go to Step 2

Remark 4 When some parameters in $\boldsymbol{p}$ describe a field (material parameter field for instance), a localization step after spatial splitting of the cost function $\mathcal{F}(\boldsymbol{p})$ can be added at the end of the first minimization (Step 2). It consists in selecting the highest local contributions to $\mathcal{F}(\boldsymbol{p})$ and updating first the associated parameters. Moreover, a goaloriented version of the model updating with mCRE, in which only parameters which have influence for the prediction of an output of interest are updated, can be constructed [48].

The mCRE formulation is thus based on a trade-off between modeling and measurement errors, which enables it to be less sensitive to noise. It inherits all the convenient properties of the CRE concept; it can be in particular extended to complex constitutive models [involving e.g. (visco)-plasticity or damage] and leads to a natural regularization. 
Notice to conclude that the mCRE strategy, without adding particular techniques, is costly. In particular, the iterative strategy requires to compute optimal admissible fields $(\widehat{u}, \widehat{\boldsymbol{q}})$ at each iteration, i.e. each time $\boldsymbol{p}_{0}$ is updated. This can be highly facilitated using a PGD meta-model, as shown in "Coupling PGD with mCRE in model validation" section.

\section{Coupling PGD with CRE in model verification}

In this section, we explain how PGD can be advantageously used when implementing CRE for model verification (see "Basics on the CRE concept" section).

\section{Constructing admissible flux fields with the hybrid-flux technique}

We consider the hybrid-flux (or EET) technique which enables to recover a flux field $\widehat{\boldsymbol{q}}_{h} \in$ $\mathcal{S}$ from a post-processing of the FE field $\boldsymbol{q}_{h}$, with local independent computations $[2,6,13]$. It is a domain decomposition approach that consists of two steps:

1. Step 1 construction of equilibrated tractions $\widehat{F}$ on the boundary $\partial K$ of each element $K \in \mathcal{T}_{h}$, with $\widehat{F}=g$ if $\partial K \subset \Gamma_{N}$, so that equilibration at the element level is verified:

$$
\int_{K} f+\int_{\partial K} \widehat{F}=0 \quad \forall K \in \mathcal{T}_{h}
$$

The construction of $\widehat{F}$ is based on the following prolongation condition:

$$
\int_{K}\left(\widehat{\boldsymbol{q}}_{h}-\boldsymbol{q}_{h}\right) \cdot \nabla \phi_{i}=0 \Longrightarrow \int_{\partial K} \widehat{F} \phi_{i}=\int_{K}\left(\boldsymbol{q}_{h} \cdot \nabla \phi_{i}-f \phi_{i}\right)
$$

applied to each element $K \in \mathcal{T}_{h}$ and each FE node $i$ connected to $K$; $\phi_{i}$ is the FE shape function associated to node $i$. This condition automatically yields equilibrated tractions $\widehat{F}$ and leads to the solution of local well-posed systems over patches of elements connected to each node $i$. In practice, tractions $\widehat{F}$ are found as linear combinations of functions $\phi_{i}$. All technical details on the construction of $\widehat{F}$ can be found in $[2,20]$.

2. Step 2 local construction, for given tractions $\widehat{F}$ and over each element $K \in \mathcal{T}_{h}$, of $\widehat{\boldsymbol{q}}_{h}$ solving the following Neumann problem:

$$
\begin{aligned}
-\nabla \cdot \widehat{\boldsymbol{q}}_{h} & =f \quad \text { in } K \quad \Longleftrightarrow \int_{K} \widehat{\boldsymbol{q}}_{h} \cdot \nabla v=\int_{K} f v+\int_{\partial K} \widehat{F} v \quad \forall v \in H^{1}(K) \\
\widehat{\boldsymbol{q}}_{h} \cdot \boldsymbol{n} & =\widehat{F} \quad \text { on } \partial K
\end{aligned}
$$

The solution of (24) to get $\widehat{\boldsymbol{q}}_{h \mid K}$ may be performed analytically, using polynomial functions with sufficiently high degree, provided the source term $f$ is polynomial as well [49]. In practice, an alternative approach with numerical solution is preferred. For fixed tractions $\widehat{F}$, the optimal admissible flux $\widehat{\boldsymbol{q}}_{h}$ inside each element $K$ is the one that minimizes the local error estimate on $K\left\|\mid \widehat{\boldsymbol{q}}-\boldsymbol{q}_{h}\right\| \|_{\mathcal{S}, K}$ (or equivalently $\|\mid \widehat{\boldsymbol{q}}\| \|_{\mathcal{S}, K}$ ) among all fluxes $\widehat{\boldsymbol{q}}$ verifying (24). Duality arguments show that this is equivalent to taking $\widehat{\boldsymbol{q}}_{h \mid K}=\mathcal{K} \nabla \rho$, with $\rho \in H^{1}(K)$ verifying:

$$
\int_{K} \mathcal{K} \nabla \rho \cdot \nabla v=\int_{K} f v+\int_{\partial K} \widehat{F} v \quad \forall v \in H^{1}(K)
$$

A numerical approximation of the solution of (25) (defined up to an additive constant) can be obtained using the FEM with a single finite element of high degree $p+k$, where $p$ denotes the polynomial degree used to compute $u_{h} \in \mathcal{U}_{h}$ and $k$ denotes the extra degree. Numerical studies performed in [50] showed that analyti- 
cal and numerical approaches give similar CRE error estimates choosing $k \geq 3$, even though the flux field is not rigorously equilibrated in each element $K$ with the latter approach. We consider the numerical approach in the following.

Solving (25) is in practice the most costly part in the hybrid-flux method (in particular for 3D applications), as it involves high-order elements and has to be performed for each element $K$. We wish to use the PGD technique in order to find, in an offline phase, a parameterized solution to (25), valid for any configuration of the geometry and the loading. From equilibrated tractions computed in Step 1 and with respect to problem data (material parameters, mesh geometry, ...), this PGD solution would then be directly used in the online error estimation phase for each element $K$ of the mesh.

\section{Use of the PGD to solve problems at the element level}

In the following:

- We consider that the material behavior is isotropic and that material parameters are constant over each element $K$, so that their values have no influence on $\widehat{\boldsymbol{q}}_{h \mid K}$; we thus set $\mathcal{K}=\mathbb{I}$ when solving (25) and define $\widehat{\boldsymbol{q}}_{h \mid K}=\nabla \rho$. In cases where $\mathcal{K}$ is not constant over each element, its evolution could be parameterized and additional material parameters would be introduced in the PGD decomposition;

- We consider, as an illustrative example, the case of 3-node triangle elements (Fig. 1). Nevertheless, the proposed strategy is generic (based on element shape functions and nodes coordinates alone) and can be straightforwardly applied to other elements.

On each edge $\Gamma^{j l}$ between vertices $j$ and $l$ of any element $K$, tractions are linear combinations of $\mathrm{FE}$ shape functions and thus read, for the considered element type, $\widehat{F}^{j l}(\boldsymbol{x})=\widehat{F}_{j}^{j l} \phi_{j}(\boldsymbol{x})+\widehat{F}_{l}^{j l} \phi_{l}(\boldsymbol{x})$ with $\left(\widehat{F}_{j}^{j l}, \widehat{F}_{l}^{j l}\right) \in \mathbb{R}^{2}$. Consequently, the solution $\rho$ to (25) can be written as a linear combination of elementary solutions:

$$
\rho(\boldsymbol{x})=\sum_{(j, l)}\left[\widehat{F}_{j}^{j l} \rho_{j}^{j l}(\boldsymbol{x})+\widehat{F}_{l}^{j l} \rho_{l}^{j l}(\boldsymbol{x})\right]
$$

where $\rho_{\ell}^{j l}(\ell=j, l)$ is the solution (up to a constant) to the elementary problem:

$$
\int_{K} \nabla \rho_{\ell}^{j l} \cdot \nabla v=\int_{\Gamma^{j l}} \phi_{\ell} v-\int_{K} \frac{1}{|K|}\left(\int_{\Gamma^{j l}} \phi_{\ell}\right) v \quad \forall v \in H^{1}(K)
$$

In the present case, there are 6 elementary problems.

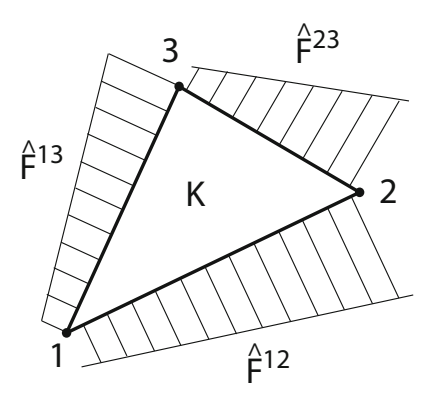

Fig. 1 Configuration at the element level. A 3-node triangle element is considered, and linear tractions $\widehat{F}^{j l}$ are defined on element edges 
Remark 5 Considering elasticity problems, (25) is changed in:

$$
\int_{K} \mathcal{K} \underline{\underline{\nabla}}^{s} \boldsymbol{\rho}: \underline{\underline{\nabla}}^{s} \boldsymbol{v}=\int_{K} \boldsymbol{f} \cdot \boldsymbol{v}+\int_{\partial K} \widehat{\boldsymbol{F}} \cdot \boldsymbol{v} \quad \forall \boldsymbol{v} \in\left[H^{1}(K)\right]^{d}
$$

where $\mathcal{K}$ is the fourth-order symmetric elasticity tensor and $\underline{\underline{\nabla}}^{s}$ is the symmetric part of the matrix gradient operator. For 3-node triangle elements, tractions on each edge $\Gamma^{j l}$ of $K \operatorname{read} \widehat{\boldsymbol{F}}^{j l}(\boldsymbol{x})=\widehat{\boldsymbol{F}}_{j}^{j l} \phi_{j}(\boldsymbol{x})+\widehat{\boldsymbol{F}}_{l}^{j l} \phi_{l}(\boldsymbol{x})$ or:

$$
\widehat{\boldsymbol{F}}^{j l}(\boldsymbol{x})=\widehat{F}_{j x}^{j l} \boldsymbol{\phi}_{j}^{x}(\boldsymbol{x})+\widehat{F}_{j y}^{j l} \boldsymbol{\phi}_{j}^{y}(\boldsymbol{x})+\widehat{F}_{l x}^{j l} \boldsymbol{\phi}_{l}^{x}(\boldsymbol{x})+\widehat{F}_{l y}^{j l} \boldsymbol{\phi}_{l}^{y}(\boldsymbol{x})
$$

with $\widehat{\boldsymbol{F}}_{\ell}^{j l}=\left(\widehat{F}_{\ell x}^{j l}, \widehat{F}_{\ell y}^{j l}\right)^{T}, \boldsymbol{\phi}_{\ell}^{x}(\boldsymbol{x})=\left(\phi_{\ell}(\boldsymbol{x}), 0\right)^{T}$ and $\boldsymbol{\phi}_{\ell}^{y}(\boldsymbol{x})=\left(0, \phi_{\ell}(\boldsymbol{x})\right)^{T}(\ell=j, l)$. We thus introduce solutions, defined up to a rigid body motion, to the following elementary problems [generalization of (27)]:

$$
\int_{K} \mathcal{K} \underline{\nabla}^{s} \boldsymbol{\rho}_{\ell}^{j l, x / y}: \underline{\nabla}^{s} \boldsymbol{v}=\int_{\Gamma^{j l}} \boldsymbol{\phi}_{\ell}^{x / y} \cdot \boldsymbol{v}-\int_{K}\left(\boldsymbol{a}_{1} \wedge \boldsymbol{X}+\boldsymbol{a}_{2}\right) \cdot \boldsymbol{v} \quad \forall \boldsymbol{v} \in\left[H^{1}(K)\right]^{2}
$$

where $\boldsymbol{X}$ are barycentric coordinates in element $K$, and $\boldsymbol{a}_{1}$ and $\boldsymbol{a}_{2}$ are defined as:

$$
\boldsymbol{a}_{1}=\frac{\left(\int_{\Gamma^{j l}} \boldsymbol{X} \wedge \boldsymbol{\phi}_{\ell}^{x / y}\right) \cdot \boldsymbol{z}}{\int_{K} \boldsymbol{X} \cdot \boldsymbol{X}} \boldsymbol{z} ; \quad \boldsymbol{a}_{2}=\frac{1}{|K|} \int_{\Gamma^{j l}} \boldsymbol{\phi}_{\ell}^{x / y}
$$

with $z$ the orthonormal vector to the 2D plane. The solution $\rho$ to (28) is then recovered as:

$$
\boldsymbol{\rho}(\boldsymbol{x})=\sum_{(j, l)}\left[\widehat{F}_{j x}^{j l} \rho_{j}^{j l, x}(\boldsymbol{x})+\widehat{F}_{j y}^{j l} \rho_{j}^{j l, y}(\boldsymbol{x})+\widehat{F}_{l x}^{j l} \rho_{l}^{j l, x}(\boldsymbol{x})+\widehat{F}_{l y}^{j l} \rho_{l}^{j l, y}(\boldsymbol{x})\right]
$$

The solution $\rho_{\ell}^{j l}$ to each problem (27) can be computed with the PGD technique, for any element $K$, parameterizing the geometry of $K$ with a set of parameters $\boldsymbol{p}_{\text {geo }} \in \mathcal{P}$. Following the approach described in [51-53], we reformulate the weak problem (27) by introducing a parameter-dependent mapping $\mathcal{M}\left(\boldsymbol{p}_{\text {geo }}\right): K_{\text {ref }} \rightarrow K\left(\boldsymbol{p}_{\text {geo }}\right)$ from a reference fixed element $K_{\text {ref }}$ to the geometrically parameterized element $K\left(\boldsymbol{p}_{\text {geo }}\right)$. Such a geometrical transformation then allows defining the weak problem in a tensor product space and applying the PGD method, in order to compute generic parameterized solutions $\rho_{\ell}^{j l}\left(\boldsymbol{p}_{\text {geo }}\right)$ which can be used for any element geometry.

Remark 6 In the presence of geometrical variabilities, an alternative approach described in $[54,55]$ could also be used. It consists in embedding the parameterized domain into a fixed fictitious domain.

In the present case, the mapping is defined from three parameters (Fig. 2):

- A first scaling mapping $\mathcal{M}_{1}: \bar{K} \rightarrow K$ maps a homothetic element $\bar{K}$ with diameter 1 to the actual element $K$ with diameter $\alpha$. This mapping reads:

$$
\left(\begin{array}{l}
x \\
y
\end{array}\right)=\mathbb{T}_{1}\left(\begin{array}{l}
\bar{x} \\
\bar{y}
\end{array}\right) ; \quad \mathbb{T}_{1}=\left[\begin{array}{cc}
\alpha & 0 \\
0 & \alpha
\end{array}\right]=\alpha \mathbb{I}
$$

- A second linear mapping $\mathcal{M}_{2}: K_{\text {ref }} \rightarrow \bar{K}$ maps a reference element $K_{\text {ref }}$ (rightangled isosceles triangle) to element $\bar{K}$. This mapping reads, using an isoparametric formulation: 


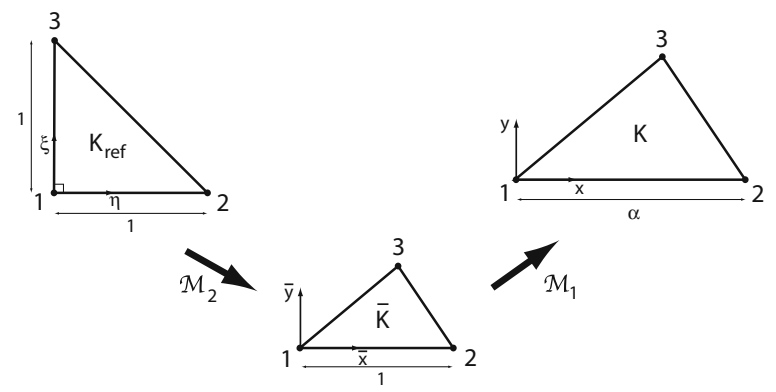

Fig. 2 Mapping between reference and parameterized elements. The mapping is defined as a combination of a scaling mapping $\mathcal{M}_{1}$ between homothetic elements $\bar{K}$ and $K$, and a linear mapping $\mathcal{M}_{2}$, defined from an isoparametric formulation, between a reference element $K_{\text {ref }}$ and $\bar{K}$

$$
\left(\begin{array}{c}
\bar{x} \\
\bar{y}
\end{array}\right)=\left(\begin{array}{c}
\phi_{2}(\eta, \xi)+\bar{x}_{3} \phi_{3}(\eta, \xi) \\
\bar{y}_{3} \phi_{3}(\eta, \xi)
\end{array}\right)=\mathbb{T}_{2}\left(\begin{array}{l}
\eta \\
\xi
\end{array}\right) ; \quad \mathbb{T}_{2}=\left[\begin{array}{cc}
1 & \bar{x}_{3} \\
0 & \bar{y}_{3}
\end{array}\right]
$$

where $\left(\bar{x}_{3}, \bar{y}_{3}\right)$ are local coordinates of node 3 in the coordinates system associated with element $\bar{K}$, and $(\eta, \xi)$ are local coordinates in the coordinates system associated with element $K_{\text {ref }}$ (see Fig. 2).

It thus involves 3 parameters and leads to the global mapping:

$$
\mathcal{M}\left(\alpha, \bar{x}_{3}, \bar{y}_{3}\right)=\mathcal{M}_{1}(\alpha) \circ \mathcal{M}_{2}\left(\bar{x}_{3}, \bar{y}_{3}\right)
$$

with transformation matrix $\mathbb{T}\left(\alpha, \bar{x}_{3}, \bar{y}_{3}\right)=\alpha \mathbb{T}_{2}\left(\bar{x}_{3}, \bar{y}_{3}\right)$, Jacobian matrix $\mathbb{J}=\mathbb{T}$, and Jaco$\operatorname{bian} J=\operatorname{det}(\mathbb{J})=\alpha^{2} \bar{y}_{3}$.

Introducing $\boldsymbol{x}_{\text {ref }}=\left(\begin{array}{l}\eta \\ \xi\end{array}\right)$, approximations of solutions $\rho_{\ell}^{j l}\left(\boldsymbol{x}_{r e f}, \alpha, \bar{x}_{3}, \bar{y}_{3}\right)$ are computed offline and once for all using the PGD technique with variable-separated modal decomposition. The parameter $\alpha$ is included only for completeness of the description; it acts as a multiplicative constant in the solution $\rho$ and disappears when computing $\widehat{\boldsymbol{q}}_{h \mid K}=\mathcal{K} \nabla \rho$. PGD solutions thus read:

$$
\rho_{\ell, m}^{j l}\left(\boldsymbol{x}_{r e f}, \alpha, \bar{x}_{3}, \bar{y}_{3}\right)=\alpha \sum_{i=1}^{m} \psi_{i}\left(\boldsymbol{x}_{r e f}\right) \delta_{i}^{x}\left(\bar{x}_{3}\right) \delta_{i}^{y}\left(\bar{y}_{3}\right)
$$

Remark 7 The number of elementary problems (27) and the number of geometrical parameters involved in the mapping $\mathcal{M}$ depend on the FE element type; for instance, 6 -node triangle elements would involve 9 elementary problems (3 for each of the three edges) and 9 geometrical parameters (12 degrees of freedom with three rigid body motions), whereas 4-node tetrahedron elements would involve 12 elementary problems ( 3 for each of the four edges) and 6 geometrical parameters (12 degrees of freedom with six rigid body motions).

\section{Implementation of the PGD}

The progressive Galerkin approach described in "Background" section is used with bilinear form $B$ and linear form $F$ constructed from the parameterized separated variable Jacobian transformation (all technical details can be found in [51,52]). Introducing the interval $I_{\alpha}$ (resp. $I_{\bar{x}_{3}}$ and $I_{\bar{y}_{3}}$ ) in which $\alpha$ (resp. $\bar{x}_{3}$ and $\bar{y}_{3}$ ) evolves, these forms read: 


$$
\begin{aligned}
& B\left(\rho_{\ell}^{j l}, v\right)=\int_{I_{\alpha}} \int_{I_{\bar{x}_{3}}} \int_{I_{\bar{y}_{3}}} \int_{K_{\text {ref }}} J \cdot \mathbb{J}^{-T} \nabla \rho_{\ell}^{j l} \cdot \mathbb{J}^{-T} \nabla v \\
& F(v)=\int_{I_{\alpha}} \int_{I_{\bar{x}_{3}}} \int_{I_{\bar{y}_{3}}}\left[\int_{\Gamma_{r e f}^{j l}} J_{s} \phi_{\ell} v-\int_{K_{r e f}} J \cdot \frac{1}{|K|}\left(\int_{\Gamma j l} \phi_{\ell}\right) v\right]
\end{aligned}
$$

Space functions $\psi_{i}\left(\boldsymbol{x}_{r e f}\right)$ in (36) are computed using the FEM with a single element of degree $p+k$. Other functions are discretized using a fine grid over spaces $I_{\alpha}, I_{\bar{x}_{3}}$, and $I_{\bar{y}_{3}}$.

Remark 8 The number $m$ of PGD modes which is required to get accurate solutions $\rho_{\ell, m}^{j l}$ can be rigorously defined using classical a posteriori error estimation tools devoted to PGD $[36,37,56,57]$. A numerical assessment of the value $m$ that yields sufficient accuracy is provided in "CRE estimate obtained from EET-PGD" section.

Remark 9 In order to save computational time and storage needs, symmetries in the local parameterized solutions $\rho_{\ell}^{j l}$ can be used. For instance, the relation $\rho_{1}^{12}\left(\eta, \xi, \alpha, \bar{x}_{3}, \bar{y}_{3}\right)=$ $\rho_{2}^{12}\left(1-\eta, \xi, \alpha, 1-\bar{x}_{3}, \bar{y}_{3}\right)$ holds.

The PGD technique thus provides for a parameterized equilibrated flux field at the element level:

$$
\begin{aligned}
& \widehat{\boldsymbol{q}}_{h, m \mid K}\left(\boldsymbol{x}_{r e f},\left\{\widehat{F}_{\ell}^{j l}\right\}, \alpha, \bar{x}_{3}, \bar{y}_{3}\right) \\
& \quad=\mathbb{J}^{-T}\left(\alpha, \bar{x}_{3}, \bar{y}_{3}\right) \nabla\left(\sum_{(j, l)}\left[\widehat{F}_{j}^{j l} \rho_{j, m}^{j l}\left(\boldsymbol{x}_{r e f}, \alpha, \bar{x}_{3}, \bar{y}_{3}\right)+\widehat{F}_{l}^{j l} \rho_{l, m}^{j l}\left(\boldsymbol{x}_{r e f}, \alpha, \bar{x}_{3}, \bar{y}_{3}\right)\right]\right)
\end{aligned}
$$

which can be directly used online in the a posteriori error estimation procedure.

Remark 10 Another study, which is not considered here, would benefit from the PGD representation $\widehat{\boldsymbol{q}}_{h, m \mid K}\left(\boldsymbol{x}_{r e f},\left\{\widehat{F}_{\ell}^{j l}\right\}, \alpha, \bar{x}_{3}, \bar{y}_{3}\right)$. It addresses the optimization of equilibrated tractions $\left\{\widehat{F}_{\ell}^{j l}\right\}$ considering a global problem in which the complementary energy is minimized. This procedure, first developed in [49], is very costly in the general case but can be highly facilitated by the explicit dependency on $\left\{\widehat{F}_{\ell}^{j l}\right\}$ provided by the PGD.

\section{Coupling PGD with mCRE in model validation}

In this section, we explain how PGD can be advantageously used when implementing mCRE for model updating (see "Extension of the CRE concept for model updating: modified CRE" section).

\section{Performing minimizations in the mCRE method}

The constrained minimization of the mCRE method (Step 2 in the algorithm given in "Extension of the CRE concept for model updating: modified CRE" section) is in practice performed finding the saddle-point of the following Lagrangian functional:

$$
\mathcal{L}(v, \boldsymbol{\pi}, \boldsymbol{p}, \lambda)=\mathcal{E}_{m C R E}^{2}(v, \boldsymbol{\pi}, \boldsymbol{p}, \boldsymbol{s})-\left[\int_{\Omega} \boldsymbol{\pi} \cdot \nabla \lambda-\int_{\Omega} f \lambda-\int_{\Gamma_{N}} g \lambda\right]
$$

for all $(v, \pi, \lambda) \in \mathcal{U} \times H(d i v, \Omega) \times \mathcal{U}$. It leads to the solution $(\widehat{u}, \widehat{\boldsymbol{q}}, \widehat{\lambda})$ of the coupled system:

$$
\int_{\Omega}(\mathcal{K}(\boldsymbol{p}) \nabla \widehat{u}-\widehat{\boldsymbol{q}}) \cdot \nabla v+\frac{r}{1-r} \sigma(\boldsymbol{\Pi} \widehat{u}-\boldsymbol{s}) \cdot \boldsymbol{\Pi} v=0 \quad \forall v \in \mathcal{U}
$$




$$
\begin{aligned}
\int_{\Omega}(\widehat{\boldsymbol{q}}-\mathcal{K}(\boldsymbol{p}) \nabla \widehat{u}) \cdot \mathcal{K}^{-1}(\boldsymbol{p}) \boldsymbol{\pi}-\int_{\Omega} \nabla \widehat{\lambda} \cdot \boldsymbol{\pi}=0 & \forall \boldsymbol{\pi} \in H(\operatorname{div}, \Omega) \\
\int_{\Omega} \widehat{\boldsymbol{q}} \cdot \nabla \lambda-\int_{\Omega} f \lambda-\int_{\Gamma_{N}} g \lambda=0 & \forall \lambda \in \mathcal{U}
\end{aligned}
$$

The second relation yields $\widehat{\boldsymbol{q}}=\mathcal{K}(\boldsymbol{p}) \nabla(\widehat{u}+\widehat{\lambda})$, and $(\widehat{u}, \widehat{\lambda})$ is obtained solving:

$$
\begin{aligned}
-\int_{\Omega} \mathcal{K}(\boldsymbol{p}) \nabla \widehat{\lambda} \cdot \nabla v+\frac{r}{1-r} \sigma \Pi \widehat{u} \cdot \Pi v & =\frac{r}{1-r} \sigma s \cdot \Pi v \quad \forall v \in \mathcal{U} \\
\int_{\Omega} \mathcal{K}(\boldsymbol{p}) \nabla(\widehat{u}+\widehat{\lambda}) \cdot \nabla \lambda & =\int_{\Omega} f \lambda+\int_{\Gamma_{N}} g \lambda \quad \forall \lambda \in \mathcal{U}
\end{aligned}
$$

The gradient of the cost function $\mathcal{F}(\boldsymbol{p})$, which is required when performing the second minimization with first order strategies, is then easily computed using the adjoint state method, as $\nabla \mathcal{F}(\boldsymbol{p})=\nabla_{\boldsymbol{p}} \mathcal{L}(\widehat{u}, \widehat{\boldsymbol{q}}, \boldsymbol{p}, \widehat{\lambda})$.

Remark 11 Usually, the discretization error is assumed to be negligible in the mCRE formulation (it can be anyway controlled using classical verification procedures, see [58]) so that a discretized version of (21) can be written using FEM, and model updating is applied directly to the discretized representation. In particular, the strong equilibrium conditions involved in the admissibility space $\mathcal{S}$ are replaced by weaker equilibrium conditions, in the FE sense only. We start from the following definitions:

- The discretized field $\boldsymbol{V}$ is KA if it verifies the (discretized) kinematic constraints of (6), so that it contains prescribed dofs. The associated admissibility space is denoted $\mathcal{U}_{h}$

- The discretized field $\boldsymbol{W}$ is SA if it verifies the FE equilibrium equations $\boldsymbol{V}^{T}(\mathbb{K} \boldsymbol{W}-\boldsymbol{F})=$ 0 for all $\boldsymbol{V} \in \mathcal{U}_{h}$, where $\mathbb{K}$ and $\boldsymbol{F}$ are the global stiffness matrix and load vector, respectively, of the FE system. The associated admissibility space is denoted $\mathcal{S}_{h}$.

The discretized mCRE functional thus reads:

$$
\mathcal{E}_{h}^{2}(\boldsymbol{V}, \boldsymbol{W}, \boldsymbol{p}, \boldsymbol{s})=\frac{1}{2}(\boldsymbol{W}-\boldsymbol{V})^{T} \mathbb{K}(\boldsymbol{p})(\boldsymbol{W}-\boldsymbol{V})+\frac{1}{2} \frac{r}{1-r}(\boldsymbol{\Pi} \boldsymbol{V}-\boldsymbol{s})^{T} \mathbb{G}(\boldsymbol{\Pi} \boldsymbol{V}-\boldsymbol{s})
$$

where $\mathbb{G}$ is a scaling diagonal matrix that integrates $\sigma$. Defining the cost function $\mathcal{F}_{h}(\boldsymbol{p})$ as:

$$
\mathcal{F}_{h}(\boldsymbol{p})=\min _{(\boldsymbol{V}, \boldsymbol{W}) \in \mathcal{U}_{h} \times \mathcal{S}_{h}} \mathcal{E}_{h}^{2}(\boldsymbol{V}, \boldsymbol{W}, \boldsymbol{p}, \boldsymbol{s})
$$

the associated constrained minimization is performed introducing the Lagrangian:

$$
\mathcal{L}_{h}(\boldsymbol{V}, \boldsymbol{W}, \boldsymbol{p}, \boldsymbol{\Lambda})=\mathcal{E}_{h}^{2}(\boldsymbol{V}, \boldsymbol{W}, \boldsymbol{p}, \boldsymbol{s})-\boldsymbol{\Lambda}^{T}[\mathbb{K}(\boldsymbol{p}) \boldsymbol{W}-\boldsymbol{F}]
$$

and leads to $(\widehat{\boldsymbol{V}}, \widehat{\boldsymbol{W}}, \widehat{\boldsymbol{\Lambda}})$ solution of the system:

$$
\begin{aligned}
\widetilde{\mathbb{K}}(\boldsymbol{p})(\widehat{\boldsymbol{U}}-\widehat{\boldsymbol{W}})+\frac{r}{1-r} \boldsymbol{\Pi}^{T} \widetilde{\mathbb{G}}(\boldsymbol{\Pi} \widehat{\boldsymbol{U}}-\boldsymbol{s}) & =\mathbf{0} \\
\mathbb{K}(\boldsymbol{p})(\widehat{\boldsymbol{W}}-\widehat{\boldsymbol{U}})-\mathbb{K}(\boldsymbol{p}) \widehat{\boldsymbol{\Lambda}} & =\mathbf{0} \\
\widetilde{\mathbb{K}}(\boldsymbol{p}) \widehat{\boldsymbol{W}}-\widetilde{\boldsymbol{F}} & =\mathbf{0}
\end{aligned}
$$


where $\widetilde{\mathbb{K}}($ resp. $\widetilde{\mathbb{G}}$ and $\widetilde{\boldsymbol{F}})$ is the restriction of $\mathbb{K}($ resp. $\mathbb{G}$ and $\boldsymbol{F}$ ) in which lines corresponding to prescribed dofs in $\mathcal{U}_{h}$ have been removed.

\section{Use of the PGD for the first minimization}

In this section, we implement a PGD meta-model to find, in an offline phase, parameterized solutions $(\widehat{u}, \widehat{\lambda})$ to (41). Defining $\sigma_{r}=\frac{r}{1-r} \sigma \in \Sigma_{r}$ (single parameter gathering scaling and weighting effects in mCRE) and assuming that $\mathcal{P}=\otimes_{j=1}^{P} \mathcal{P}_{j}$, these are searched of the form:

$$
\begin{aligned}
\widehat{u}_{m}\left(\boldsymbol{x}, \sigma_{r}, \boldsymbol{p}\right)= & \sum_{i=1}^{m}\left[\psi_{i}^{u}(\boldsymbol{x}) \kappa_{i}^{u}\left(\sigma_{r}\right) \prod_{j=1}^{P} \chi_{j, i}^{u}\left(p_{i}\right)\right] ; \\
& \widehat{\lambda}_{m}\left(\boldsymbol{x}, \sigma_{r}, \boldsymbol{p}\right)=\sum_{i=1}^{m}\left[\psi_{i}^{\lambda}(\boldsymbol{x}) \kappa_{i}^{\lambda}\left(\sigma_{r}\right) \prod_{j=1}^{P} \chi_{j, i}^{\lambda}\left(p_{i}\right)\right]
\end{aligned}
$$

Here again, the progressive Galerkin approach described in "Background" section is used with the following bilinear form $B$ and linear form $F$ :

$$
\begin{aligned}
& B((\widehat{u}, \widehat{\lambda}),(v, \lambda))=\int_{\Sigma_{r}} \int_{\mathcal{P}}\left[\int_{\Omega} \mathcal{K}(\boldsymbol{p}) \nabla(\widehat{u}+\widehat{\lambda}) \cdot \nabla \lambda-\int_{\Omega} \mathcal{K}(\boldsymbol{p}) \nabla \widehat{\lambda} \cdot \nabla v+\sigma_{r} \Pi \widehat{u} \cdot \boldsymbol{\Pi} v\right] \\
& F((v, \lambda))=\int_{\Sigma_{r}} \int_{\mathcal{P}}\left[\int_{\Omega} f \lambda+\int_{\Gamma_{N}} g \lambda+\sigma_{r} \boldsymbol{s} \cdot \Pi v\right]
\end{aligned}
$$

Using then $\left(\widehat{u}_{m}, \widehat{\lambda}_{m}\right)$ in the online model updating phase with mCRE has several advantages:

- The explicit dependency on parameters $\boldsymbol{p}$ enables: (1) to evaluate very fast and for any values of $\boldsymbol{p}$ the optimal admissible fields arising from the first constrained minimization; (2) to compute gradients of the cost function $\mathcal{F}(\boldsymbol{p})$ analytically and thus perform the second minimization step very easily;

- The explicit dependency on parameter $\sigma_{r}$ makes the definition of the optimal value of $\sigma_{r}$ (primarily with respect to measurement noise using the L-curve method) straightforward.

Remark 12 In the present work, we assume that measurement values in $\boldsymbol{s}$ are known upstream to the updating procedure, and that this procedure is conducted for a single set of measurement values. In other cases such as data assimilation on time-dependent problems, they can be considered as extra-parameters in the PGD decomposition as performed in $[40,41]$.

In practice, space functions $\psi_{i}^{u}(\boldsymbol{x})$ and $\psi_{i}^{\lambda}(\boldsymbol{x})$ are computed using the FEM, and other functions appearing in PGD modes are discretized using a fine grid over spaces $\Sigma_{r}$ and $\mathcal{P}_{j}$ $(j=1, \ldots, P)$.

\section{Results and discussion}

In this section, we illustrate and analyze performances of the approach proposed in "Coupling PGD with CRE in model verification" and "Coupling PGD with mCRE in model validation" sections. "Example 1: a posteriori error estimation on a 2D structure" section deals with model verification using a CRE error estimate coupled with PGD, whereas 
"Example 2: model updating on a 3D structure" section addresses model updating using a mCRE formulation coupled with PGD.

\section{Example 1: a posteriori error estimation on a 2D structure}

Problem geometry and data

We consider discretization error estimation on a $2 \mathrm{D}$ holed plate $\Omega$, according to a given mesh composed of 3-nodes triangular elements (Fig. 3). We consider a steady-state thermal problem and homogeneous isotropic material properties with $\mathcal{K}=\mathbb{I}$. A prescribed zero temperature is applied on the external boundary $\Gamma_{D}$, while a flux $g=1 \mathrm{~W} / \mathrm{m}$ is imposed on the inner boundary $\Gamma_{N}$. Owing to problem symmetries, only one quarter of the plate is studied.

From the associated FE solution, equilibrated tractions are computed using the first step of the hybrid-flux (or EET) technique.

\section{Details on the PGD solution}

We compute a parametrized solution $\rho_{\ell, m}^{j l}\left(\boldsymbol{x}_{\text {ref }}, \alpha, \bar{x}_{3}, \bar{y}_{3}\right)=\alpha \sum_{i=1}^{m} \psi_{i}\left(\boldsymbol{x}_{\text {ref }}\right) \delta_{i}^{x}\left(\bar{x}_{3}\right) \delta_{i}^{y}\left(\bar{y}_{3}\right)$ of (25) with a single 4 th order FE element and 20 PGD modes $(m=20)$. The domains $I_{\bar{x}_{3}}=[0,1]$ and $I_{\bar{y}_{3}}=[0.1,1]$ are discretized with 100 points each, after checking that this is sufficient to ensure an accurate description of the evolutions with respect to $\bar{x}_{3}$ and $\bar{y}_{3}$. The first three PGD modes of $\rho_{1,20}^{11}$ are shown in Fig. 4. In Fig. 5, we represent the PGD approximation of $\rho_{1}^{11}$ for different configurations of parameters $\bar{x}_{3}$ and $\bar{y}_{3}$. The computation of this PGD solution is done once for all, in an offline phase and stored for later use.

After identifying the PGD parameters $\alpha, \bar{x}_{3}$ and $\bar{y}_{3}$ over each element of the mesh (see Fig. 6), an accurate PGD approximation of the admissible flux $\widehat{\boldsymbol{q}}_{m}$ can then be directly evaluated inside each element in an inexpensive online phase; this method is referred as EET-PGD method in the following.

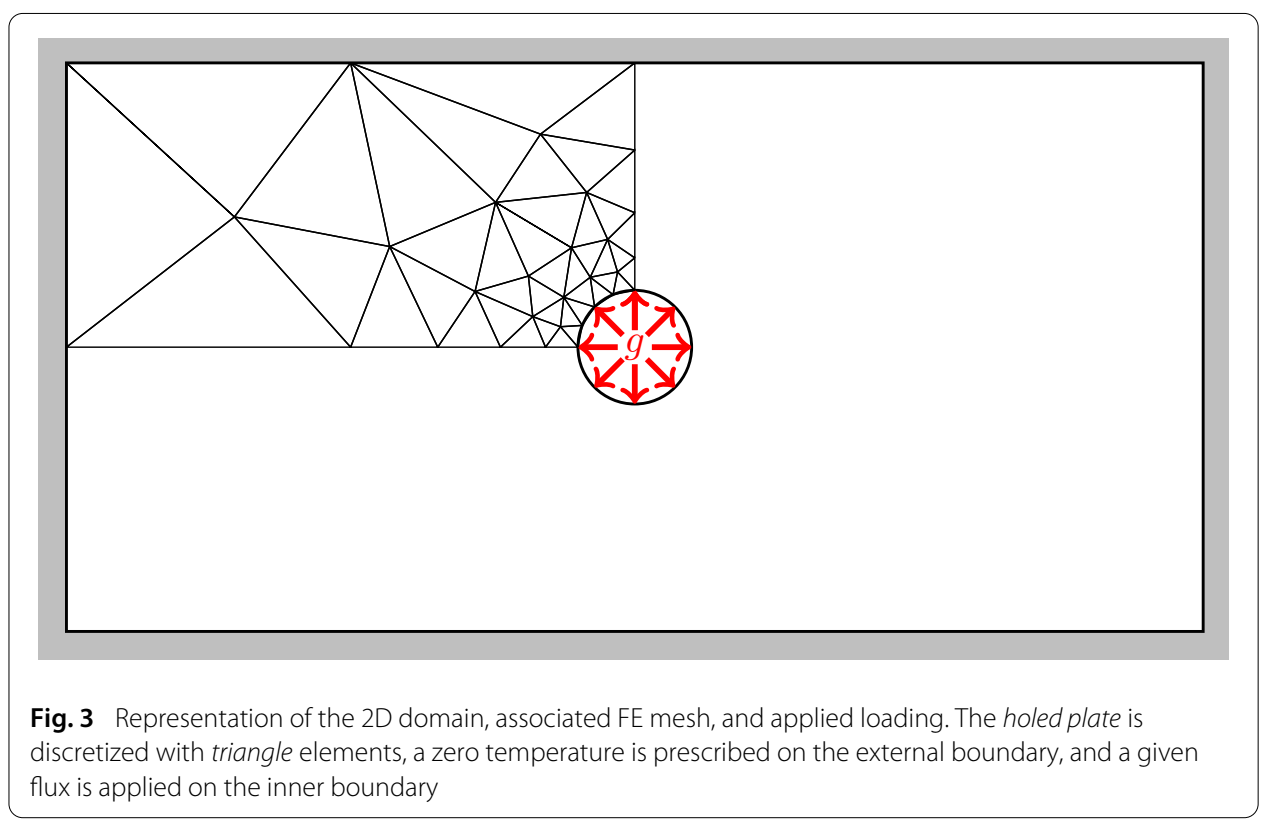



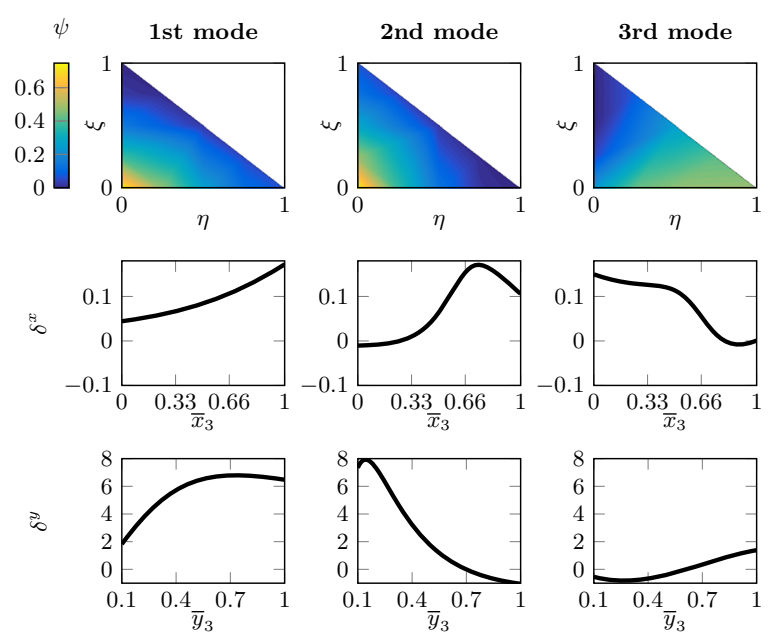

Fig. 4 First PGD modes of $\rho_{1,20}^{11}$. Space functions $\psi_{i}$ (top), and parameters functions $\delta_{i}^{x}$ (center) and $\delta_{i}^{y}$ (bottom) are represented with respect to the order $i$, for $i=1,2,3$ (from left to right)

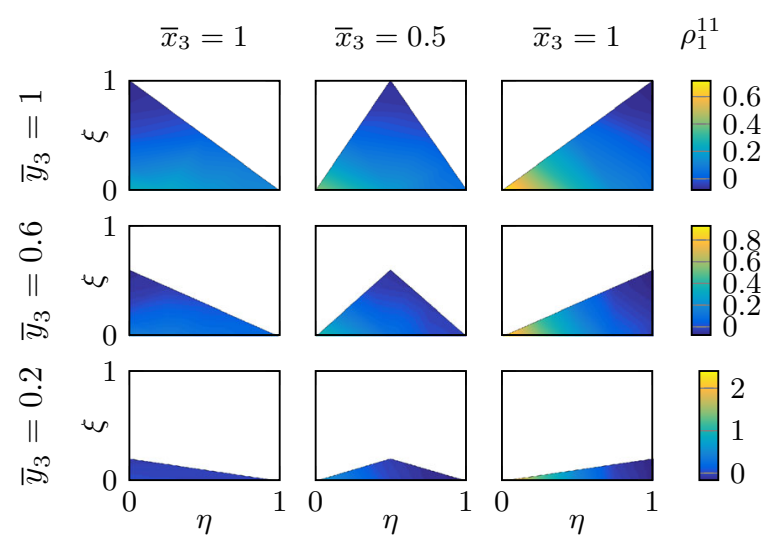

Fig. 5 PGD solution $\rho_{1,20}^{11}$. The parameterized approximate solution is represented over the element $\bar{K}$ for various parameter configurations

\section{CRE estimate obtained from EET-PGD}

From PGD solutions, we have all ingredients to estimate the discretization error using the CRE method. In Fig. 7, we compare local contributions to the CRE estimate $2 E_{C R E}^{2}$, obtained from the EET-PGD technique when computing an admissible flux $\widehat{\boldsymbol{q}}_{h, m}$, with: (1) contributions to the CRE estimate obtained from the classical EET technique when computing an admissible flux $\widehat{\boldsymbol{q}}_{h}$; (2) contributions to the exact error $\|\mid e\| \|_{\mathcal{U}}^{2}$ evaluated using a highly refined mesh (overkill solution). One observes similarities between the two CRE estimations, showing up areas where the mesh needs to be refined. These areas are correctly predicted when comparing to the exact error distribution.

Choosing $m=20$ to compute PGD solutions in the EET-PGD technique may be unnecessary. To analyze this point, we show in Fig. 8 values of the effectivity index $i_{\text {eff }}=\frac{\sqrt{2} E_{C R E}}{\|\mid\| e \|_{\mathcal{U}}}$ with respect to the number $m$ of PGD modes used to evaluate the equilibrated flux $\widehat{\boldsymbol{q}}_{h, m}$. We also represent in Fig. 9 the evolution of the relative error $\frac{\left\|\widehat{\boldsymbol{q}}_{h, m}-\widehat{\boldsymbol{q}}_{\|}\right\| \|_{\mathcal{S}}}{\left\|\widehat{\boldsymbol{q}}_{h}\right\| I_{\mathcal{S}}}$ with respect to 

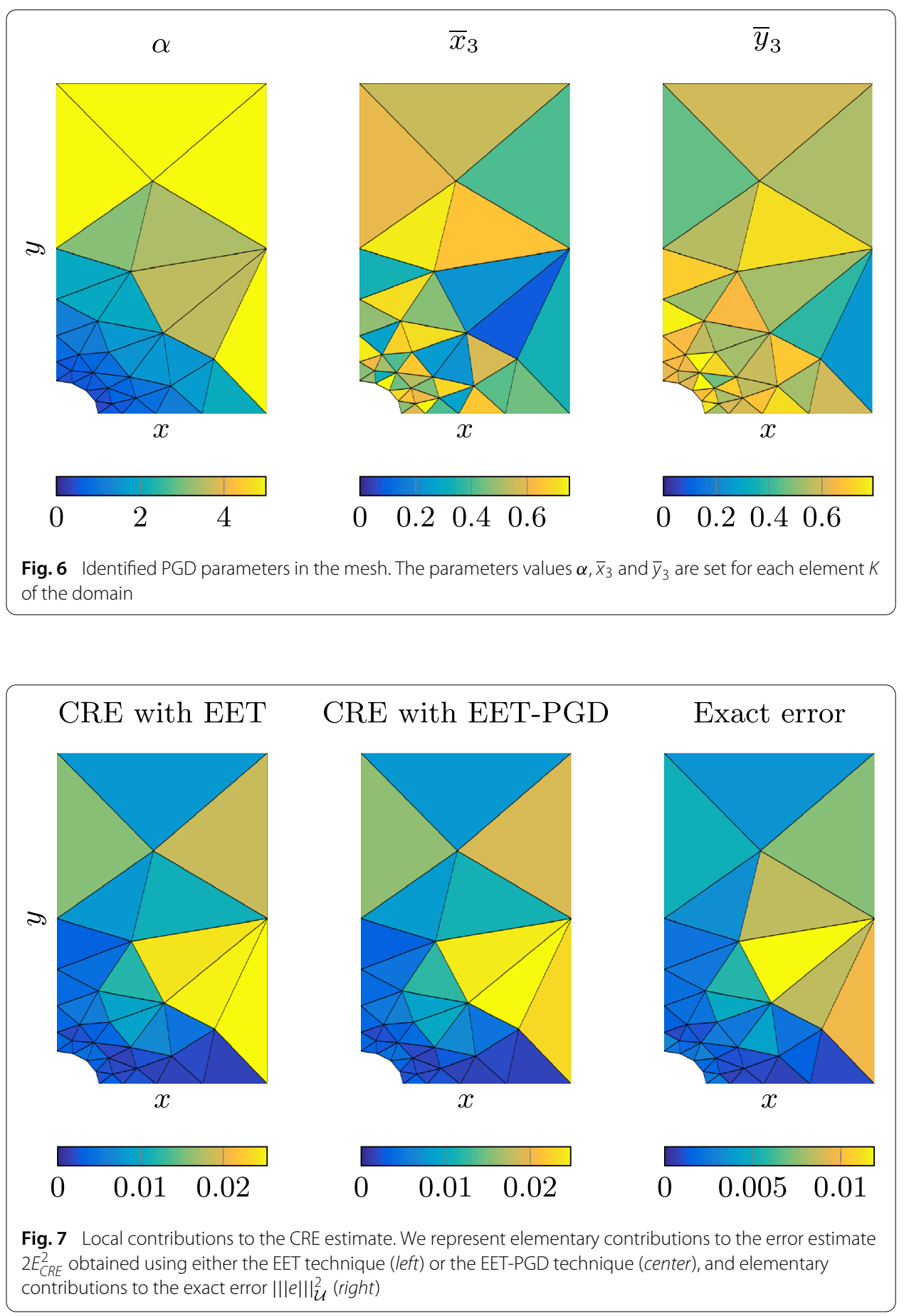

$m$, where $\widehat{\boldsymbol{q}}_{h}$ is the equilibrated flux field constructed with the EET technique. A map of \|\|$\widehat{\boldsymbol{q}}_{h, m}-\widehat{\boldsymbol{q}}_{h} \|_{\mathcal{S}}$ for $m=1, m=2$, and $m=3$ is given in Fig. 10 . We observe that choosing $m=7$ is enough to reconstruct an admissible flux solution which is equivalent to the one obtained with the classical EET technique. We also observe that $m=3$ enables to capture the complexity of the local problems and to provide for a relevant error estimate, even though it is not guaranteed. 


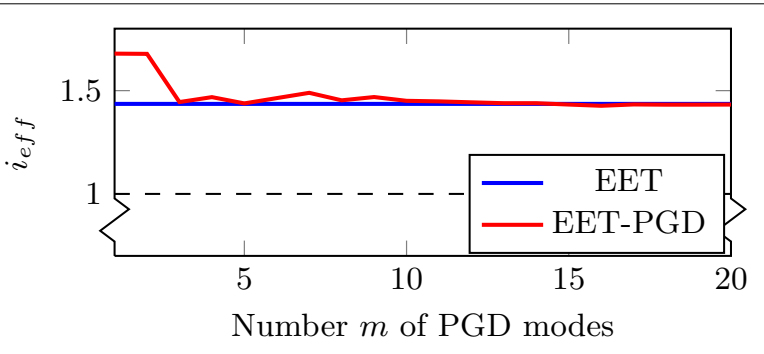

Fig. 8 Influence on the effectivity index. We represent the value of the effectivity index with respect to the number of PGD modes used to evaluate the equilibrated flux in the CRE estimate

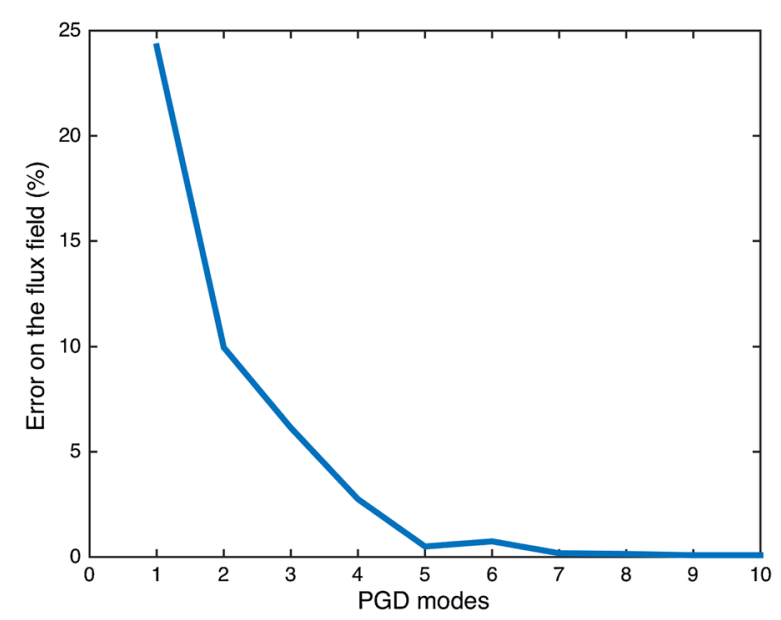

Fig. 9 Error on the PGD construction. We represent the evolution of the relative error $\left\|\left|\widehat{\boldsymbol{q}}_{h, m}-\widehat{\boldsymbol{q}}_{h}\right|\right\|_{\mathcal{S}} /\left\|\left|\widehat{\boldsymbol{q}}_{h}\right|\right\|_{\mathcal{S}}$ with respect to the number of PGD modes used to compute $\widehat{\boldsymbol{q}}_{h, m}$
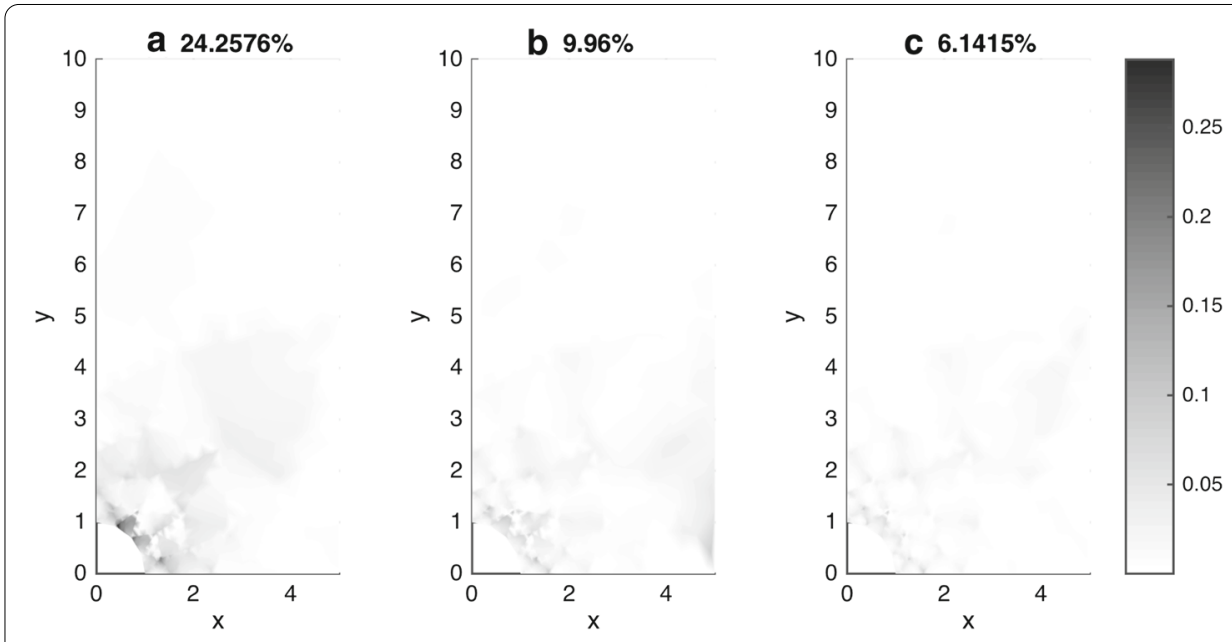

Fig. 10 Map of the error on the flux field computed with PGD. The spatial distribution of the error $\left\|\mid \widehat{\boldsymbol{q}}_{h, m}-\widehat{\boldsymbol{q}}_{h}\right\| \|_{\mathcal{S}}$ is represented for $m=1$ (left), $m=2$ (center), and $m=3$ (right)

\section{Speed-up obtained using the PGD solution}

Eventually, we compare the CPU time required to compute the equilibrated flux field depending on which method is used (Fig. 11). All the computations were performed on an 


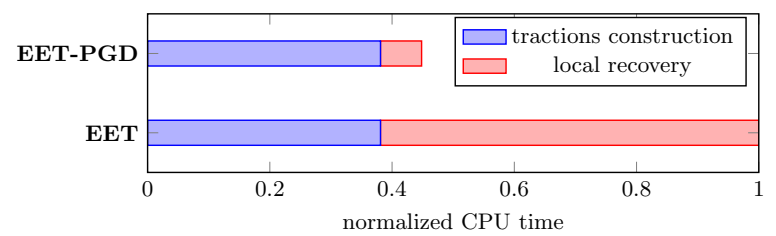

Fig. 11 Comparison of CPU times. Values of CPU times required to compute the hybrid-flux steps are given depending on the equilibrated flux technique which is used

Intel Core i5 2.4 GHz with $8 \mathrm{~GB}$ of RAM, without parallelization. Classical EET and EETPGD techniques share as much code as possible, and only the construction and solution of the matrix problem is replaced by a simple post-processing with PGD solutions in the EET-PGD technique. Naturally, the first step with construction of equilibrated tractions is similar for both techniques.

When using the EET-PGD technique, the offline CPU cost to compute the PGD solution is $312 \mathrm{~s}$; this solution can then be used in a multi-query context. In the online step, computing the equilibrated flux from the classical EET technique (Cholesky factorization) takes $0.01509 \mathrm{~s}$ per element ( $0.0587 \mathrm{~s}$ for the whole mesh composed of 42 elements), whereas computing the equilibrated flux from a direct evaluation of the PGD solution takes $0.00426 \mathrm{~s}$ per element $(0.0077 \mathrm{~s}$ for the whole mesh). We thus observe a speed-up of almost 10 in the second CRE step (construction of equilibrated fluxes in each element), and the global speed-up on the whole hybrid-flux technique (with associated CPU cost of $0.0960 \mathrm{~s})$ is about a factor 2 .

In Fig. 12, we represent this same speed-up for different levels of refinement of the initial mesh (corresponding meshes are given in Fig. 13). The speed-up increases as the mesh becomes finer, reaching a gain of 125 on a 2688 elements mesh for the local recovery, while the overall hybrid-flux technique shows a speed-up of magnitude 5 on this same mesh.

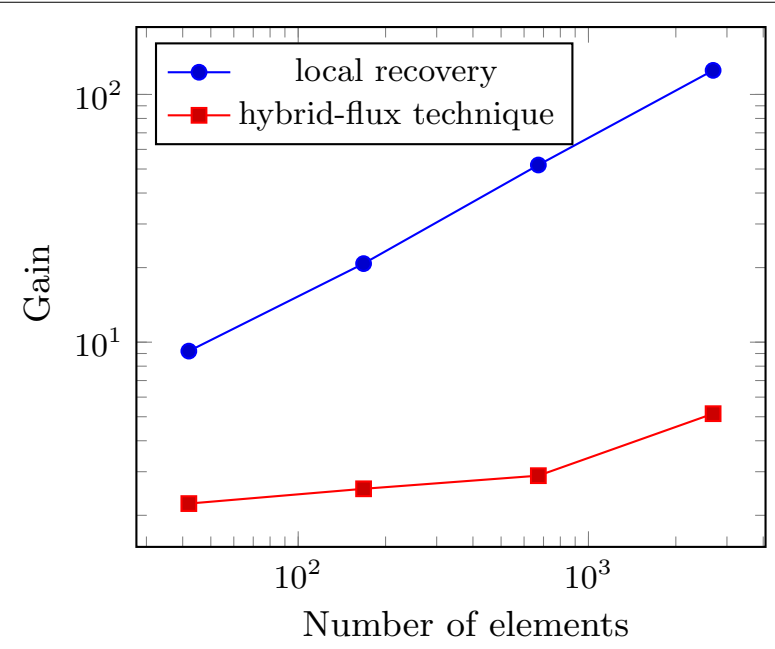

Fig. 12 CPU gains. For meshes with increasing numbers of elements, the CPU gains on the equilibrated flux step are given. Gains on the local recovery alone or on the overall hybrid-flux technique are explicited 


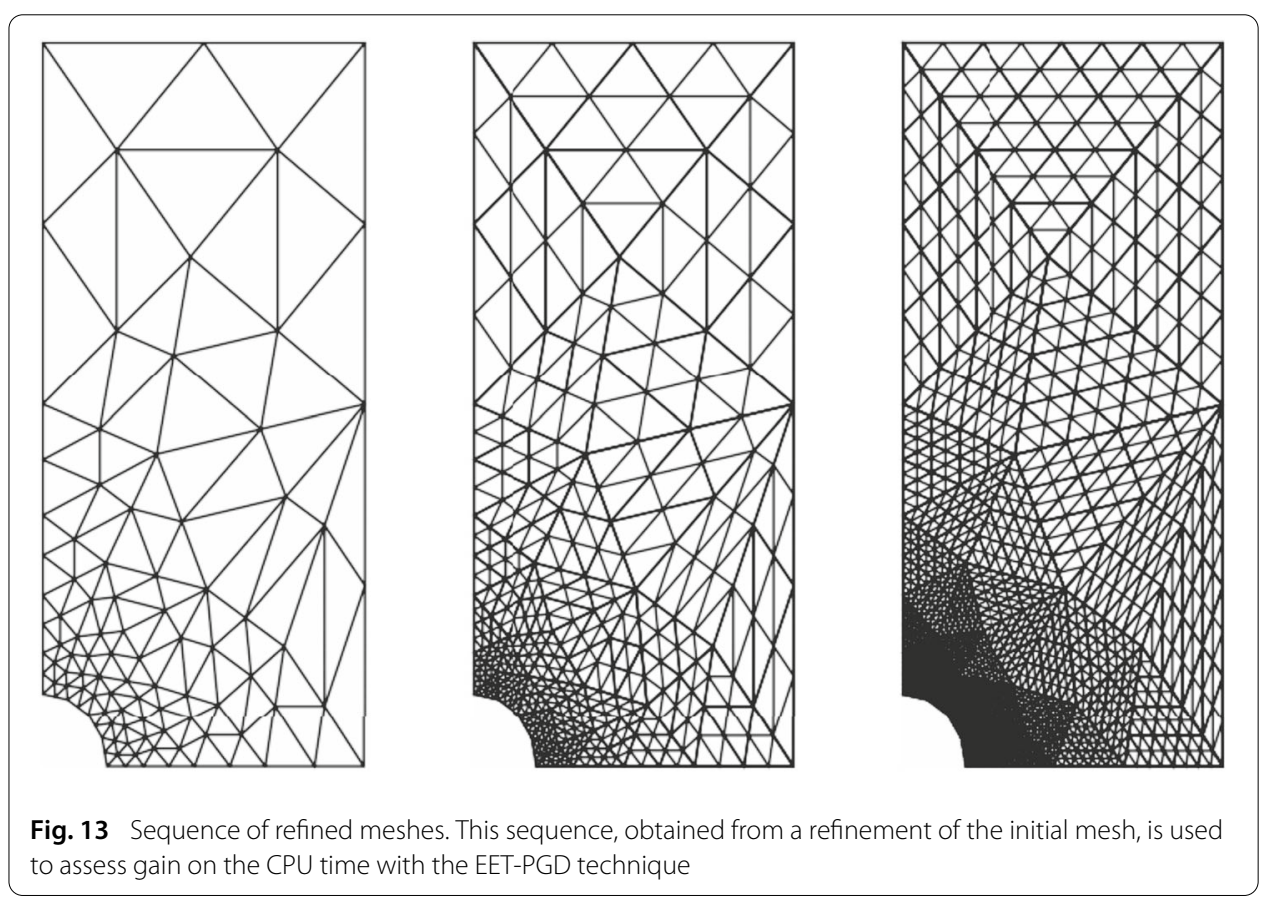

An additional step would be to reduce the CPU time of the tractions reconstruction, by optimizing implementation, in order to fully benefit from the use of the PGD technique.

\section{Example 2: model updating on a 3D structure Identification problem}

We consider a steady-state thermal problem on the 3D geometry shown in Fig. 14. It is a two layers cylinder (length $L=100$, internal radius $R_{\text {int }}=10$, external radius $R_{\text {ext }}=14$ ) with a localized inclusion (length $L_{i n c}=10$ ) in the middle of the cylinder. The internal layer (resp. external layer, and inclusion) is represented in green (resp. blue, and red) color in Fig. 14. In each of the layers and in the inclusion, the material is supposed to be isotropic and homogeneous with respective material operators $\mathcal{K}_{\text {int }}=p_{\text {int }} \mathbb{I}, \mathcal{K}_{\text {ext }}=p_{\text {ext }} \mathbb{I}$, and $\mathcal{K}_{i n c}=p_{i n c} \mathbb{I}$. The applied boundary conditions are: (1) homogeneous Dirichlet boundary conditions on one end of the cylinder; (2) given thermal flow $q^{d}=1$ on the inner boundary; (3) zero thermal flow (free surface) on all other boundaries.

We wish to identify thermal conductivity parameters $\left(p_{\text {ext }}, p_{\text {inc }}\right)$ from noisy measurements given by a set of 12 sensors. These sensors are placed on four horizontal rows with $\pi / 6$ angle spacing (see Fig. 14). The reference values for parameters $\left(p_{\text {ext }}, p_{\text {inc }}\right)$ to be identified are $p_{\text {ext }}^{r e f}=10$ and $p_{i n c}^{r e f}=1$. Furthermore, we fix $p_{i n t}=20$.

To perform the identification process, the structure is discretized with a FE mesh made of 41,856 tetrahedra $(13,164$ nodes) as presented in Fig. 14. The noisy observation data are synthesized numerically by solving the direct problem with reference parameter values $\left(p_{\text {ext }}^{r e f}, p_{\text {inc }}^{r e f}\right)$, extracting the obtained nodal temperature values $u_{i}$ at sensors positions, then adding a Gaussian white noise to get data $s_{i}$ :

$$
s_{i}=(1+\mathcal{N}(0, \Upsilon)) u_{i}
$$

with variance $\Upsilon$. In the following, we choose $\Upsilon=0.1$. 


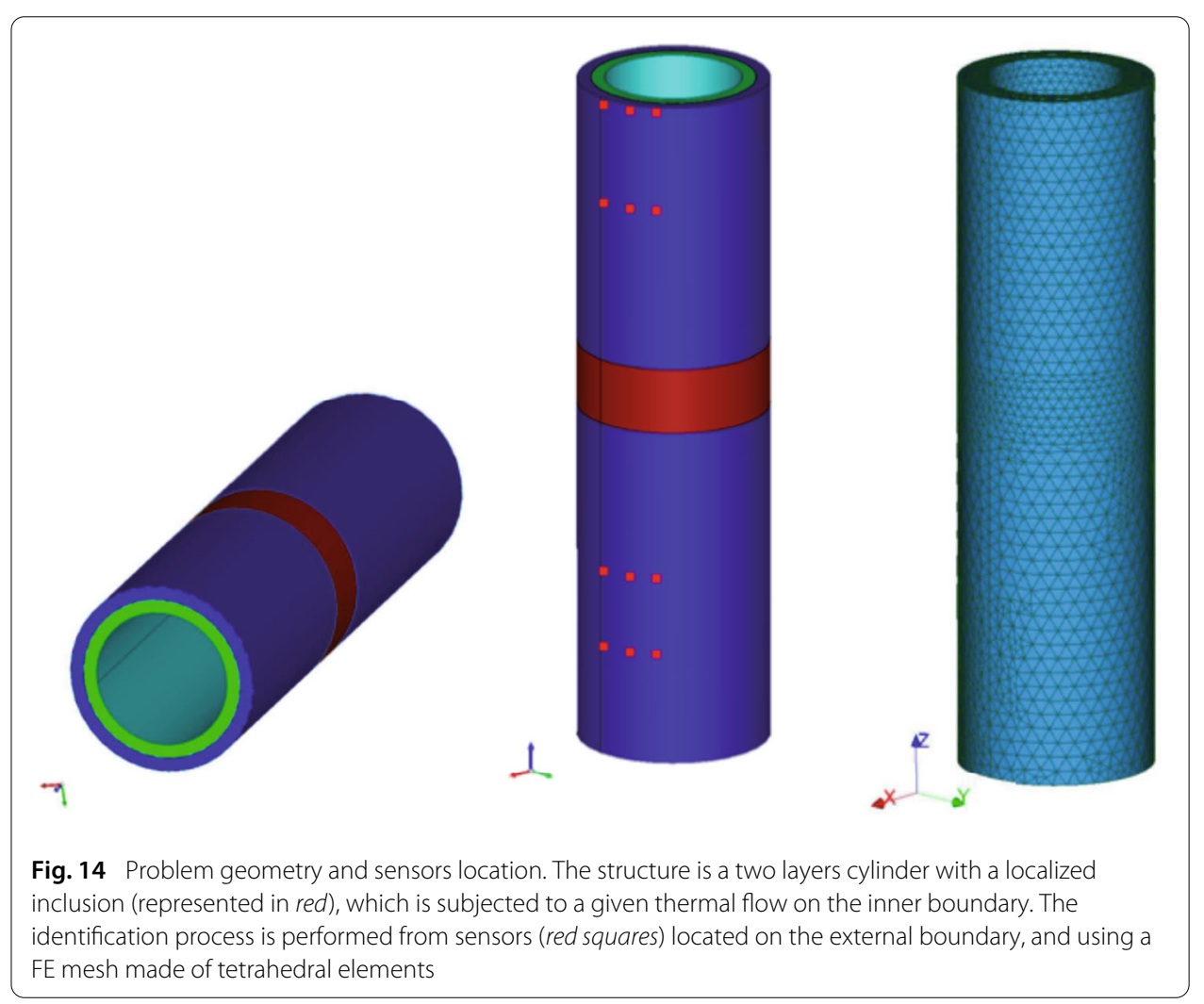

\section{PGD model reduction}

As detailed in "Coupling PGD with mCRE in model validation" section, PGD representations of the parameterized solutions $(\widehat{u}, \widehat{\lambda})$ to $(41)$ are computed in an offline phase. These read:

$$
\begin{aligned}
& \widehat{u}_{m}\left(\boldsymbol{x}, \sigma_{r}, p_{\text {ext }}, p_{\text {inc }}\right)=\sum_{i=1}^{m} \psi_{i}^{u}(\boldsymbol{x}) \kappa_{i}^{u}\left(\sigma_{r}\right) \chi_{1, i}^{u}\left(p_{\text {ext }}\right) \chi_{2, i}^{u}\left(p_{\text {inc }}\right) \\
& \widehat{\lambda}_{m}\left(\boldsymbol{x}, \sigma_{r}, p_{\text {ext }}, p_{\text {inc }}\right)=\sum_{i=1}^{m} \psi_{i}^{\lambda}(\boldsymbol{x}) \kappa_{i}^{\lambda}\left(\sigma_{r}\right) \chi_{1, i}^{\lambda}\left(p_{\text {ext }}\right) \chi_{2, i}^{\lambda}\left(p_{\text {inc }}\right)
\end{aligned}
$$

The first five (normalized) PGD modes are represented in Figs. 15 and 16. In Fig. 17, we represent the energy norm of each PGD mode relative to the energy norm of the first PGD mode $u_{1}$, which shows that their influence highly decreases with $m$. In practice, we choose $m=15$.

\section{Identification with PGD}

Starting from the initial parameter values $p_{\text {ext }}^{0}=20$ and $p_{i n c}^{0}=10$, we implement the model updating process using the mCRE method coupled with the previously computed PGD solutions. We represent in Fig. 18 the evolution of the cost function $\mathcal{F}\left(p_{\text {ext }}, p_{\text {inc }}\right)=$ $\mathcal{E}_{m C R E}^{2}\left(\widehat{u}_{m}, \widehat{\boldsymbol{q}}_{m}, p_{\text {ext }}, p_{i n c}, \boldsymbol{s}\right)$; it clearly shows the convex feature of this cost function, and therefore the uniqueness of the minimization solution. We also plot in Fig. 19 the evolution of the two terms of the cost function, i.e. the model error term and the measurement error term, with respect to the penalty coefficient $\sigma_{r}$ for $\left(p_{\text {ext }}, p_{\text {inc }}\right)=\left(p_{\text {ext }}^{0}, p_{i n c}^{0}\right)$. The optimal value of $\sigma_{r}$ is the one for which the two error terms are balanced (i.e. when the two curves 


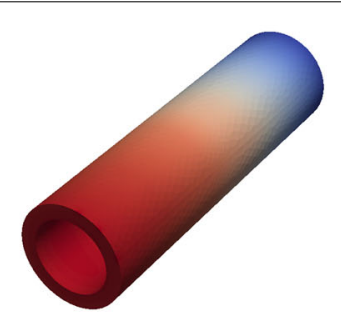

a $\psi_{1}^{u}$

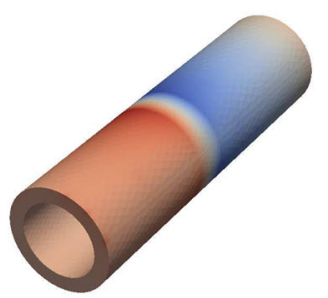

c $\psi_{2}^{u}$

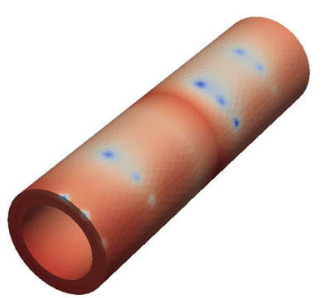

e $\psi_{3}^{u}$

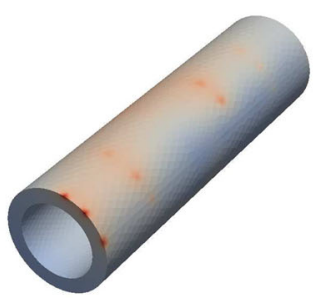

g $\psi_{4}^{u}$

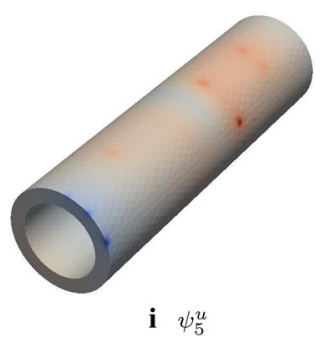

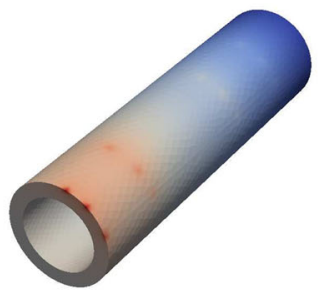

b $\psi_{1}^{\lambda}$

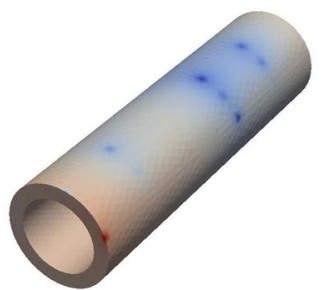

d $\psi_{2}^{\lambda}$

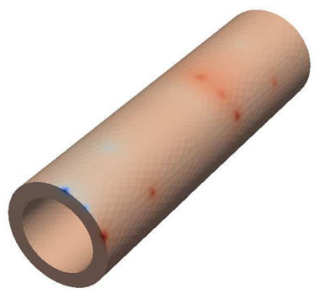

f $\psi_{3}^{\lambda}$

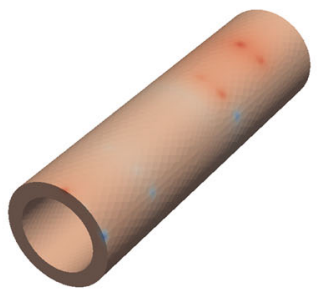

h $\psi_{4}^{\lambda}$

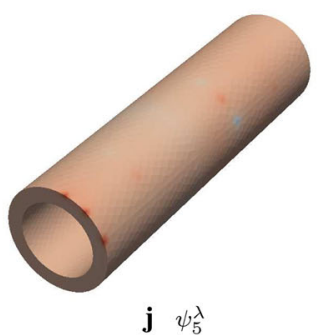

Fig. 15 Spatial PGD modes for the identification with mCRE. The first five (normalized) modes for the primal field (left), and the adjoint field (right), are plotted

intersect). Notice that these evolutions of the mCRE functional are easy to obtain as the PGD solutions (49) lead to explicit dependencies with respect to $p_{\text {ext }}, p_{i n c}$, and $\sigma_{r}$.

We now perform the iterative process using a first order (gradient) minimization method. For each iteration, we show in Fig. 20 the identified values of $\left(p_{\text {ext }}, p_{\text {inc }}\right)$, as 


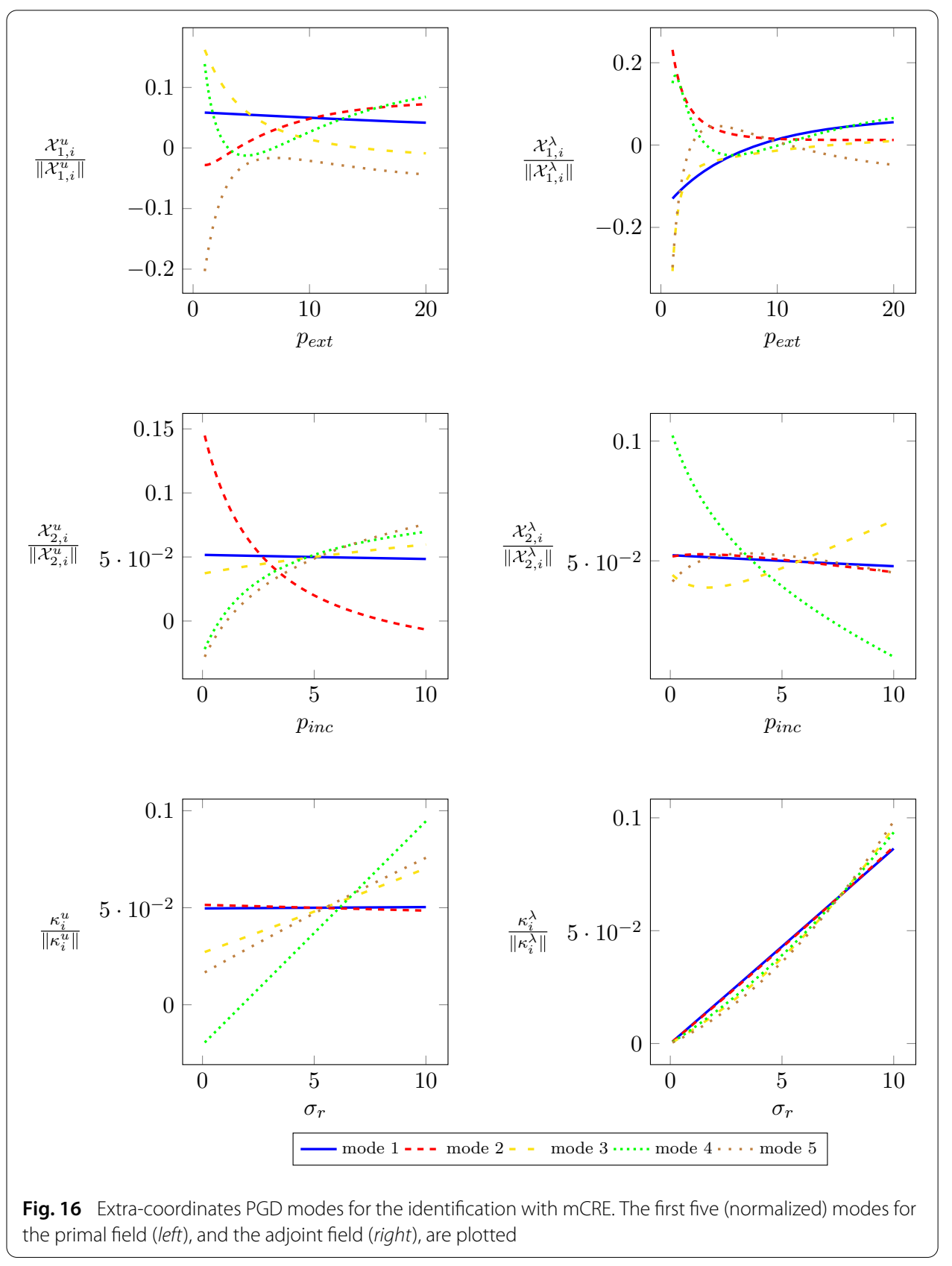

well as the optimal value $\sigma_{r}$ used for this iteration and defined as previously. We observe that the method converges to identified values of $\left(p_{\text {ext }}, p_{\text {inc }}\right)$ which are very close to the reference values $\left(p_{\text {ext }}^{r e f}, p_{i n c}^{r e f}\right)$. In addition, we study the incidence of the number $m$ of considered PGD modes on the identification results. The convergence of the identification process is represented in Fig. 21 for several values of $m$. We clearly observe that the accuracy of the identification results is highly impacted by the value chosen for $m$, and that the process leads to a relative error lower than $10 \%$ for both parameters $p_{\text {ext }}$ and $p_{\text {inc }}$ when using $m=15$. It is also interesting to notice that the PGD representation with $m=10$ is suitable for the identification of $p_{\text {ext }}$, which is the parameter with greater weight on the overall solution, but still fails for the identification of $p_{\text {inc }}$. 

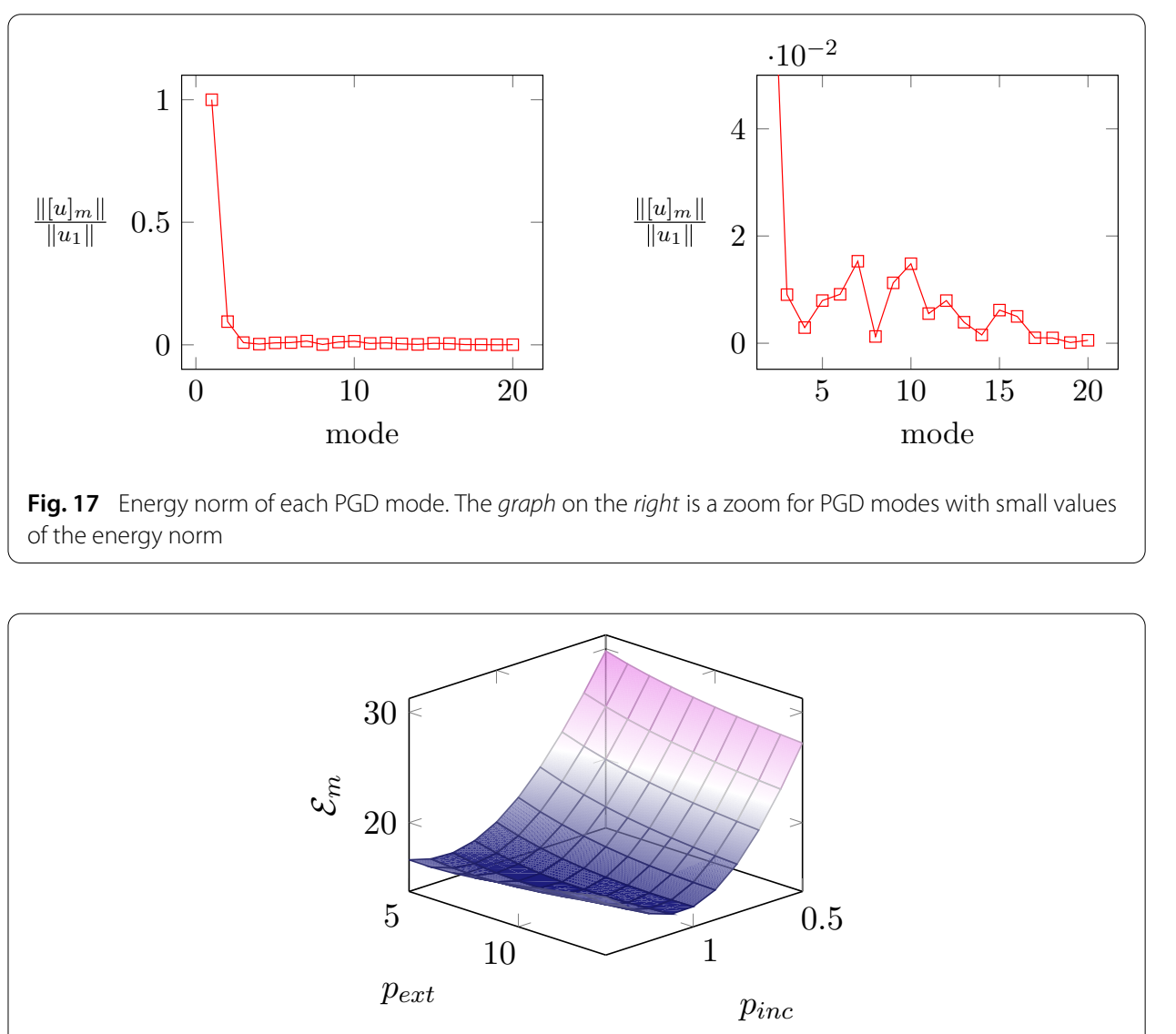

Fig. 18 Convex shape of the mCRE cost function. We represent the evolution of the mCRE functional with respect to $p_{\text {ext }}$ and $p_{\text {inc }}$

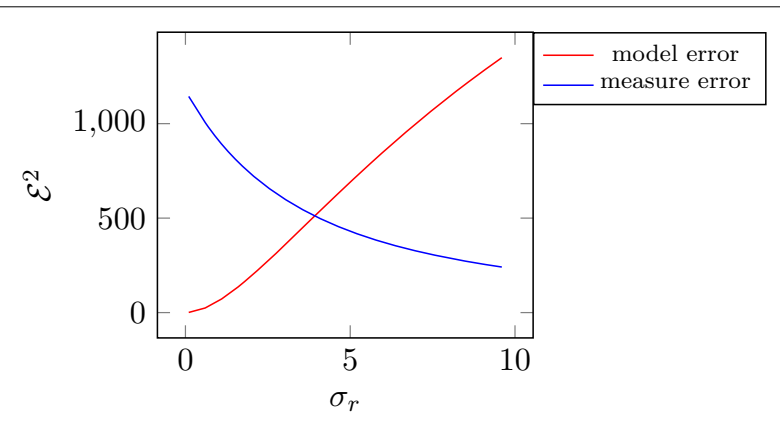

Fig. 19 Trade-off in the mCRE functional. We represent the evolution of the two terms which compose the $\mathrm{mCRE}$ functional, i.e. the model error term and the measurement error term, with respect to $\sigma_{r}$

The use of PGD enables large computation gains. Using a direct solver with parallelization over 4 nodes, a classical identification process with mCRE would require about $4 \mathrm{~h}$ for this problem. Coupled with PGD, this same process takes only 5 min in the online phase (and additional 30 min to compute PGD solutions with 15 modes in the offline phase). All computations were performed with a Python FE code using the scipy.sparse module for matrix representation, and systems were solved with a dedicated direct solver based on the UMFPACK library. The speed-up thus comes from the difference between 


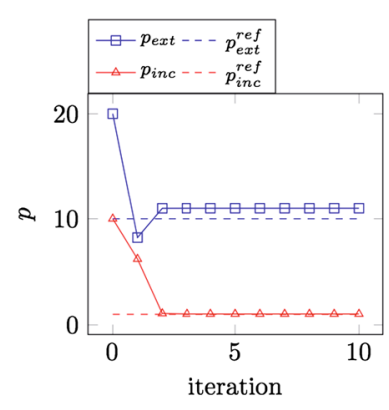

a Identification results

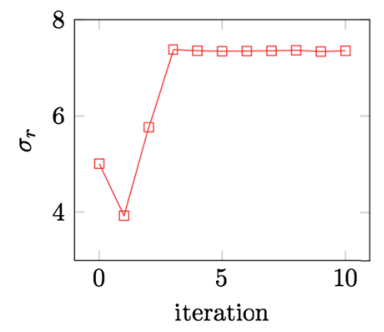

b Penalty coefficient evolution

Fig. 20 Identification results. Identified values of parameters $p_{\text {ext }}$ and $p_{\text {inc }}(l e f t$ ), and optimal value of the penalty coefficient $\sigma_{r}(r i g h t)$, are given with respect to iterations
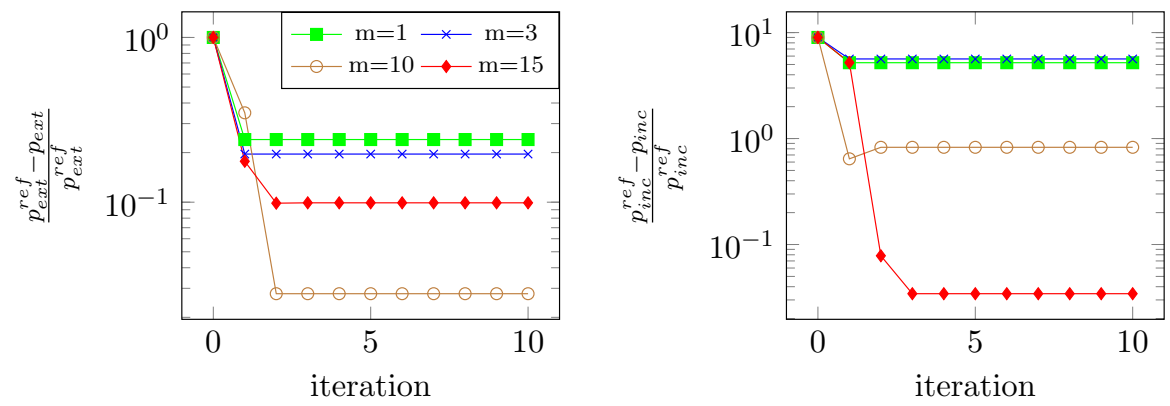

Fig. 21 Influence of $m$ on the identification results. We represent the relative error on the identified values of parameters $p_{\text {ext }}$ (left) and $p_{\text {inc }}$ (right), along the identification iterations, with respect to the value of $m$

the original mCRE and the PGD-mCRE strategies. A crude complexity analysis of the two approaches can be conducted as follows:

- Considering original mCRE, each iteration with update of the value of $\sigma_{r}$ requires to solve a Pareto problem to find the optimal value of $\sigma_{r}$. This involves about 40 sub-iterations, each of them corresponding to the solution of a linear system with the size of the problem in space [resulting from (41)]. Considering 10 iterations in the mCRE identification process thus leads to the solution of about 400 linear systems of the space problem size;

- Considering PGD-mCRE, the offline computational cost is due to the use of a greedy algorithm to compute 15 PGD modes. At each iteration of this algorithm, we implement a fixed point procedure which converges in 3 sub-iterations (average), and a sub-iteration requires the solution of the space problem. Consequently, the computation of the parametric PGD decomposition requires to solve about 45 linear systems with the size of the problem in space. Then, no more solutions of linear systems are required in the online step, merely some inexpensive evaluations of parametric functions.

\section{Conclusions}

We presented a general framework that highlights the beneficial use of PGD in V\&V procedures performed by means of the CRE concept. Based on an offline/online strategy, 
it drastically decreases the computational cost and technicalities which are essentially associated with the computation of admissible fields. We believe this work paves the way to both robust, practical, and real-time methods for controlling computational mechanics models. Furthermore, as the proposed technique is focused on balance equations alone, it should be possible to extend it to nonlinear time-dependent problems. This will be the topic of forthcoming research works.

\section{Authors' contributions}

All authors discussed the content of the article, and were involved in its writing. All authors read and approved the final manuscript.

\section{Competing interests}

The authors declare that they have no competing interests.

Received: 7 February 2016 Accepted: 19 May 2016

Published online: 03 June 2016

\section{References}

1. Babuška I, Strouboulis T. The finite element method and its reliability. Oxford: Oxford University Press; 1999.

2. Ladevèze $P$, Pelle J-P. Mastering calculations in linear and nonlinear mechanics. New York: Springer; 2004.

3. Chamoin L, Díez P, editor. Verifying calculations, forty years on: an overview of classical verification techniques for FEM simulations. Berlin: Springer; 2015.

4. Bonnet M, Constantinescu A. Inverse problems in elasticity. Inverse Problems. 2005;21(2):R1.

5. Ladevèze P. Comparaison de modèles de milieux continus. Thèse d'état, Université P. et M. Curie, Paris; 1975.

6. Ladevèze $P$, Leguillon D. Error estimate procedure in the finite element method and application. SIAM J Numer Anal. 1983;20(3):485-509.

7. Destuynder P, Métivet B. Explicit error bounds in a conforming finite element method. Math Comput. 1999;68(288):1379-96.

8. Ladevèze $P$, Moës N. A new a posteriori error estimation for nonlinear time-dependent finite element analysis. Comput Methods Appl Mech Eng. 1998;157:45-68.

9. Ladevèze P. Constitutive relation error estimations for finite element analyses considering (visco)-plasticity and damage. Int J Numer Methods Eng. 2001;52(5-6):527-42.

10. Ladevèze $P$. Strict upper error bounds for calculated outputs of interest in computational structural mechanics. Comput Mech. 2008;42(2):271-86.

11. Ladevèze $P$, Chamoin L. Calculation of strict error bounds for finite element approximations of nonlinear pointwise quantities of interest. Int J Numer Methods Eng. 2010;84:1638-64.

12. Ladevèze P, Pled F, Chamoin L. New bounding techniques for goal-oriented error estimation applied to linear problems. Int J Numer Methods Eng. 2013;93(13):1345-80.

13. Ladevèze $P$, Maunder EAW. A general method for recovering equilibrating element tractions. Comput Methods Appl Mech Eng. 1996;137:111-51.

14. Cottereau R, Díez P, Huerta A. Strict error bounds for linear solid mechanics problems using a subdomain based flux-free method. Comput Mech. 2009;44(4):533-47.

15. Gallimard L. A constitutive relation error estimator based on traction-free recovery of the equilibrated stress. Int J Numer Methods Eng. 2009;78(4):460-82.

16. Moitinho de Almeida JP, Maunder EAW. Recovery of equilibrium on star patches using a partition of unity technique. Int J Numer Methods Eng. 2009;79:1493-516.

17. Parès N, Santos H, Díez P. Guaranteed energy error bounds for the Poisson equation using a flux-free approach: solving the local problems in subdomains. Int J Numer Methods Eng. 2009;79:1203-44.

18. Ern A, Vohralik M. A posteriori error estimation based on potential and flux reconstruction for the heat equation. SIAM J Numer Anal. 2010;345(48):198-223.

19. Ladevèze $P$, Chamoin L, Florentin $E$. A new non-intrusive technique for the construction of admissible stress fields in model verification. Comput Methods Appl Mech Eng. 2010;199(9-12):766-77.

20. Pled F, Chamoin L, Ladevèze $P$. On the techniques for constructing admissible stress fields in model verification: performances on engineering examples. Int J Numer Methods Eng. 2011;88(5):409-41.

21. Ladevèze $P$, Nedjar D, Reynier M. Updating of finite element models using vibration tests. AIAA J. 1994;32(7):1485-91.

22. Chouaki A, Ladevèze $P$, Proslier $L$. An updating of structural dynamic model with damping. Inverse problems in engineering: theory and practice; 1996. p. 335-42

23. Ladevèze $P$, Chouaki $A$. Application of a posteriori error estimation for structural model updating. Inverse Probl. 1999;15(1):49-58.

24. Bonnet $M$, Aquino W. Three-dimensional transient elastodynamic inversion using the modified error in constitutive relation. Inverse Probl. 2015;31(3):035010.

25. Bui HD, Constantinescu A. Spatial localization of the error of consitutive law for the identification of defects in elastic bodies. Arch Mech. 2000;52:511-22.

26. Ladevèze $P$, Puel $G$, Deraemaeker $A$, Romeuf $T$. Validation of structural dynamics models containing uncertainties. Comput Methods Appl Mech Eng. 2006;195(4-6):373-93.

27. Faverjon B, Ladevèze P, Louf F. Validation of stochastic linear structural dynamics models. Comput Struct. 2009;87(1314):829-37. 
28. Allix $\mathrm{O}$, Feissel $\mathrm{P}$, Nguyen $\mathrm{H}$. Identification strategy in the presence of corrupted measurements. Eng Comput. 2005;22(5-6):487-504.

29. Feissel P, Allix O. Modified constitutive relation error identification strategy for transient dynamics with corrupted data: the elastic case. Comput Methods Appl Mech Eng. 2007;196(13-16):1968-83.

30. Florentin $\mathrm{E}$, Lubineau $\mathrm{G}$. Identification of the parameters of an elastic material model using the constitutive equation gap method. Comput Mech. 2010;46:521-31.

31. Ben Azzouna M, Feissel P, Villon P. Robust identification of elastic properties using the modified constitutive relation error. Comput Methods Appl Mech Eng. 2015;295:196-218.

32. Chinesta F, Ammar A, Cueto E. Recent advances and new challenges in the use of the proper generalized decomposition for solving multidimensional models. Arch Comput Methods Eng. 2010;17(4):327-50.

33. Chinesta $F$, Ladevèze $P$, Cueto $E$. A short review on model order reduction based on proper generalized decomposition. Arch Comput Methods Eng. 2011;18:395-404.

34. Chinesta F, Keunings R, Leygue A. The proper generalized decomposition for advanced numerical simulations. Berlin: Springer International Publishing; 2014.

35. Nouy A. A priori model reduction through proper generalized decomposition for solving time dependent partial differential equations. Comput Methods Appl Mech Eng. 2010;199:1603-26.

36. Ladevèze $P$, Chamoin $L$. On the verification of model reduction methods based on the proper generalized decomposition. Comput Methods Appl Mech Eng. 2011;200:2032-47.

37. Ladevèze P, Chamoin L. Toward guaranteed PGD-reduced models. In: Zavarise G, Boso DP, editors. Bytes and science. Barcelona: CIMNE; 2012.

38. Allier $P E$, Chamoin $L$, Ladevèze P. Proper generalized decomposition omputational methods on a benchmark problem: introducing a new strategy based on constitutive relation error minimization. Adv Model Simul Eng Sci. 2015;2(1):1-25.

39. Gonzalez D, Masson F, Poulhaon F, Leygue A, Cueto E, Chinesta F. Proper generalized decomposition based dynamic data driven inverse identification. Math Comput Simul. 2012;82(9):1677-95.

40. Bouclier R, Louf F, Chamoin L. Real-time validation of mechanical models coupling PGD and constitutive relation error. Comput Mech. 2013;52(4):861-83.

41. Marchand B, Chamoin L, Rey C. Real-time updating of structural mechanics models using Kalman filtering, modified Constitutive Relation Error and Proper Generalized Decomposition. Accepted International Journal in Numerical Methods in Engineering. 2016. doi:10.1002/nme.5197.

42. Fraeijs de Veubeke B, Hugge MA. Dual analysis for heat conduction problems by finite elements. Int I Numer Methods Eng. 1972;5(1):65-82.

43. Oden JT, Reddy JN. On dual complementary variational principles in mathematical physics. Int J Eng Sci. 1974;12:1-29.

44. Fraeijs de Veubeke B. Displacement and equilibrium models in the finite element method. Int I Numer Methods Eng Class Repr Ser. 2001;52:287-342.

45. Chavent G, Kunisch K, Roberts J. Primal-dual formulations for parameter estimation problems. Comput Appl Math. 1999;18:173-229.

46. Hansen P. Rank-deficient and discrete ill-posed problems. Philadelphia: SIAM; 1998.

47. Deraemaeker A, Ladevèze P, Romeuf T. Model validation in the presence of uncertain experimental data. Eng Comput. 2004;21(8):808-33.

48. Chamoin L, Ladevèze P, Waeytens J. Goal-oriented updating of mechanical models using the adjoint framework. Comput Mech. 2014;54(6):1415-30.

49. Ladevèze $P$, Rougeot $P$. New advances on a posteriori error on constitutive relation in finite element analysis. Comput Methods Appl Mech Eng. 1997;150:239-49.

50. Babuška I, Strouboulis T, Upadhyay CS, Gangaraj SK, Copps K. Validation of a posteriori error estimators by numerical approach. Int J Numer Methods Eng. 1994;374(7):1073-123.

51. Ammar A, Huerta A, Leygue A, Chinesta F, Cueto E. Parametric solutions involving geometry: a step towards efficient shape optimization. Comput Methods Appl Mech Eng. 2014;268(1):178-93.

52. Zlotnik S, Díez P, Modesto D, Huerta A. Proper generalized decomposition of a geometrically parametrized heat problem with geophysical applications. Int J Numer Methods Eng. 2015;103(10):737-58.

53. Courard A, Néron D, Ladevèze P, Ballère L. Integration of PGD-virtual charts into an engineering design process. Comput Mech. 2015.

54. Canuto C, Kozubek T. A fictitious domain approach to the numerical solution of pdes in stochastic domains. Numer Math. 2007;107(2):257-93.

55. Nouy A, Chevreuil M, Safatly E. Fictitious domain method and separated representations for the solution of boundary value problems on uncertain parameterized domains. Comput Methods Appl Mech Eng. 2011;200:3066-82.

56. Ammar A, Chinesta F, Díez P, Huerta A. An error estimator for separated representations of highly multidimensional models. Comput Methods Appl Mech Eng. 2010;199(25-28):1872-80.

57. Moitinho de Almeida JP. A basis for bounding the errors of proper generalised decomposition solutions in solid mechanics. Int J Numer Methods Eng. 2013;94(10):961-84.

58. Becker $R$, Vexler B. Mesh refinement and numerical sensitivity analysis for parameter calibration of partial differential equations. J Comput Phys. 2005;206:95-110. 